FEDERAL RESERVE BANK OF SAN FRANCISCO

WORKING PAPER SERIES

\title{
Term Structure Analysis with Big Data
}

\author{
Martin M. Andreasen \\ Aarhus University \\ Jens H. E. Christensen \\ Federal Reserve Bank of San Francisco \\ Glenn D. Rudebusch \\ Federal Reserve Bank of San Francisco
}

September 2017

Working Paper 2017-21

http://www.frbsf.org/economic-research/publications/working-papers/2017/21

\section{Suggested citation:}

Andreasen, Martin M., Jens H. E. Christensen, Glenn D. Rudebusch. 2017. “Term Structure Analysis with Big Data” Federal Reserve Bank of San Francisco Working Paper 2017-21. https://doi.org/10.24148/wp2017-21

The views in this paper are solely the responsibility of the authors and should not be interpreted as reflecting the views of the Federal Reserve Bank of San Francisco or the Board of Governors of the Federal Reserve System. 


\title{
Term Structure Analysis with Big Data
}

\author{
Martin M. Andreasen ${ }^{\dagger}$ \\ Jens H. E. Christensen ${ }^{\ddagger}$ \\ Glenn D. Rudebusch*
}

\begin{abstract}
Analysis of the term structure of interest rates almost always takes a two-step approach. First, actual bond prices are summarized by interpolated synthetic zero-coupon yields, and second, a small set of these yields are used as the source data for further empirical examination. In contrast, we consider the advantages of a one-step approach that directly analyzes the universe of bond prices. To illustrate the feasibility and desirability of the onestep approach, we compare arbitrage-free dynamic term structure models estimated using both approaches. We also provide a simulation study showing that a one-step approach can extract the information in large panels of bond prices and avoid any arbitrary noise introduced from a first-stage interpolation of yields.
\end{abstract}

JEL Classification: C55, C58, G12, G17

Keywords: extended Kalman filter, fixed-coupon bond prices, arbitrage-free Nelson-Siegel model

We thank participants at the Big Data in Dynamic Predictive Econometric Modeling Conference and the 10th Annual SoFiE Conference for helpful comments. The views in this paper are solely the responsibility of the authors and should not be interpreted as reflecting the views of the Federal Reserve Bank of San Francisco or the Federal Reserve System. We thank Patrick Shultz for outstanding research assistance.

${ }^{\dagger}$ Department of Economics, Aarhus University, Denmark; e-mail: mandreasen@econ.au.dk.

${ }^{\ddagger}$ Corresponding author: Federal Reserve Bank of San Francisco, 101 Market Street MS 1130, San Francisco, CA 94105, USA; phone: 1-415-974-3115; e-mail: jens.christensen@sf.frb.org.

*Federal Reserve Bank of San Francisco; e-mail: glenn.rudebusch@sf.frb.org.

This version: September 15, 2017. 


\section{Introduction}

Most term structure analysis takes a two-step approach to examining prices of fixed-income securities. First, a set of constant-maturity zero-coupon yields are constructed from a sample of bond prices, and then these synthetic yields are used as the input to estimate the dynamic term structure model (DTSM) of interest. In the past, this separation was especially convenient because of the computational burden of working with large data sets of actual bond prices. Indeed, the widespread popularity of the two-step approach has implied that the estimation of these synthetic zero-coupon yields typically is taken for granted and given little consideration, despite the challenges documented in the construction of these synthetic yields in, e.g., Bliss (1996) and Gürkaynak et al. (2007, 2010). Furthermore, some researchers such as Dai et al. (2004) and Fontaine and Garcia (2012) have argued that synthetic interpolated yields can erase interesting bond pricing information by excessive smoothing and may even introduce unnecessary measurement error to the data.

The contribution of the present paper is to show that the initial step of constructing synthetic zero-coupon yields can be avoided, as progress in computing power now allows term structure analysis to work directly with the big data universe of bond prices. Indeed, we document that standard DTSMs can be reliably estimated via a one-step approach using a large panel of observed bond prices. We illustrate this alternative to the conventional twostep approach by comparing identical DTSMs that are estimated by the one-step and two-step approaches - both using an empirical sample of bond prices and simulated bond prices in a Monte Carlo study. ${ }^{1}$

Our empirical application focuses on the Canadian government bond market between January 2000 and April 2016, which is chosen because its size is representative of sovereign bond markets in many developed countries. In addition, Canadian bonds face no appreciable credit risk during our sample, and these bonds are not materially affected by liquidity issues and safety premiums on recently-issued securities, which plague analysis of U.S. Treasuries. ${ }^{2}$ In total, our Canadian sample for the one-step approach contains end-of-month prices on 105 bonds. The corresponding data for the two-step approach follows the existing literature and uses a limited number of synthetic zero-coupon yields. We consider two sources for such synthetic yields. The first data set is produced by the Bank of Canada and described in Bolder et al. (2004). We construct the second data set of synthetic yields by estimating the flexible

\footnotetext{
${ }^{1}$ Duffee (1999), Driessen (2005), Fontaine and Garcia (2012), and Pancost (2017) also estimate DTSMs on actual bond prices, but they do not compare their results to those obtained from the corresponding two-step approach as done in the present paper.

${ }^{2}$ In the creation of interpolated nominal U.S. Treasury yield curves, Gürkaynak et al. (2007) generally exclude the two most recently issued securities, i.e. the "on-the-run" and "first off-the-run" bonds, which often trade at a premium. A one-step approach could also exclude these bond prices or augment the DTSM of interest to accommodate bond-specific liquidity characteristics as in Fontaine and Garcia (2012) and Andreasen et al. (2017), among others.
} 
parametric discount function of Svensson (1995) on the same panel of bond prices as used for the one-step approach. ${ }^{3}$ The differences between these two data sets of synthetic zerocoupon yields are generally small for maturities within the one- to ten-year maturity range, but the differences may easily exceed ten basis points outside this maturity range where fewer bonds are available. This observation provides tentative evidence that the various curvefitting techniques used to construct synthetic zero-coupon yields may induce nonnegligible measurement errors in these yields.

We then estimate the same DTSMs on Canadian bond prices via the one-step and twostep approaches using either synthetic zero-coupon yields from the Bank of Canada or from the Svensson (1995) yield curve. Our benchmark DTSM is the arbitrage-free Nelson-Siegel (AFNS) model of Christensen et al. (2011), which is a Gaussian affine model where level, slope, and curvature factors explain the evolution of the yield curve. We highlight two findings from estimating this model on our Canadian sample. First, the parameters that determine the functional form between bond yields and the latent factors (i.e. the risk-neutral parameters) are those most affected by the choice of estimation approach. For instance, the decay parameter $\lambda$ in the AFNS model, which determines how the slope and curvature factor affect bond yields, varies notably. Thus, the parameters in a DTSM can be affected by using synthetic zero-coupon yields as opposed to the underlying market prices. Second, we also show that the proposed one-step approach gives a substantially closer fit to the underlying coupon bonds than the conventional two-step approach. For instance, the ability of the AFNS model to fit the market prices of coupon bonds may reduce the root mean squared fitted errors by as much as $44 \%$ when the model is estimated by the proposed one-step approach instead of the conventional two-step approach. This shows that the use of synthetic yields in the two-step approach may add some noise to the predicted bond prices from an estimated DTSM.

We also demonstrate how to address the inherent nonlinearities when pricing coupon bonds in most DTSMs and implement the one-step approach with maximum likelihood estimation of model parameters and latent factors. Furthermore, to show the general applicability of the proposed one-step approach, we estimate a nonlinear DTSM that enforces the zero-lower bound, and a five-factor model to get an even tighter fit of long-term Canadian bonds than implied by our benchmark three-factor model.

As a supplement to these empirical estimates, we also explore the finite-sample properties of the proposed one-step approach and the conventional two-step approach in a Monte Carlo study. A novel feature of this simulation experiment is to work at the level of coupon bonds and hence account for estimation uncertainty in the construction of synthetic zero-coupon

\footnotetext{
${ }^{3}$ Other functional forms could be considered such as the cubic splines used by Steeley (2008), the hybrid combination of cubic splines and parametric functions advocated by Faria and Almaida (2017), or the optimally smooth spline yield curves derived from an exact bootstrap method based on the Moore-Penrose pseudoinverse developed by Filipović and Willems (2016).
} 
yields within the two-step approach. The main insight from this Monte Carlo study is that DTSMs may be estimated more reliably by directly estimating them on observed bond prices instead of synthetic zero-coupon yields. Although these synthetic zero-coupon yields are estimated very accurately with well-established curve-fitting techniques, we nevertheless find that seemingly negligible errors in these synthetic yields do affect the estimated parameters in a DTSM. In particular, all risk-neutral parameters are estimated with smaller biases and greater efficiency in the proposed one-step approach compared with the conventional two-step approach.

The remainder of the paper is structured as follows. Section 2 describes the Canadian government bond data, while Section 3 briefly summarizes the AFNS model and presents its estimation results on Canadian data. Section 4 provides several extensions of the analysis in Section 3, while Section 5 is devoted to our Monte Carlo study. We provide an out-ofsample forecasting exercise of the Canadian three-month yield in Section 6, before concluding in Section 7. Appendices contain additional details on the characteristics of the Canadian government bonds, our construction of synthetic zero-coupon yields based on the Svensson (1995) yield curve, the model estimation, and formulas for yield decompositions.

\section{The Canadian Bond Market}

This section describes the market for Canadian government bonds. We first describe our sample of Canadian bonds for the one-step approach in Section 2.1, before presenting two data sets of synthetic zero-coupon yields for the two-step approach in Section 2.2.

\subsection{The Universe of Government Bonds}

As of April 2016, the Canadian government bond market had a total outstanding notional amount of CAD 512.5 billion, which is equivalent to $25 \%$ of Canadian GDP. The Canadian government holds a AAA rating with a stable outlook by all major rating agencies, meaning that no correction for credit risk is required. The number of individual fixed-coupon bonds in our sample is shown in panel (a) of Figure 1. The number of bonds grows gradually from about 15 bonds at the start of the sample to roughly 45 bonds in 2012, where it has remained until the end of our sample in 2016.

The time-varying maturity distribution of all 105 bonds in our sample is illustrated in panel (b) of Figure 1, where each security is represented by a downward-sloping line showing its remaining years to maturity at each date. Since two-year bonds are issued several times each year, the short end of the fixed-coupon bond market has remained densely populated at all times. As for medium-term maturities, five-year bonds were issued once a year between 2000 and 2006, were halted in 2007 and 2008, and made semi-annual since 2009. There has also 


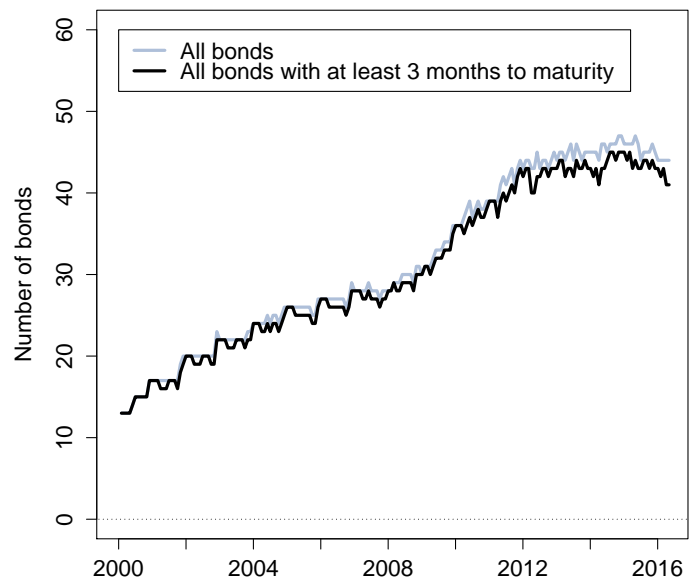

(a) Number of Canadian bonds

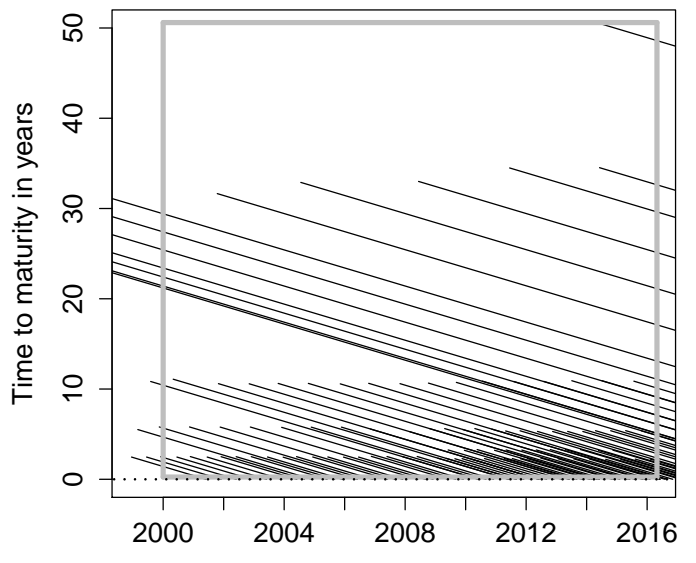

(b) Maturity distribution of Canadian bonds

Figure 1: Description of The Canadian Bond Market

Panel (a) shows the number of Canadian government bonds at each date. The solid grey line refers to the entire sample of bonds. The solid black line indicates the number of bonds when eliminating bonds with less than three months to maturity. Panel (b) shows the maturity distribution of the Canadian government bonds considered. The grey rectangle indicates the subsample used throughout the paper.

been a regular issuance of ten-year bonds once a year since the start of our sample. Finally, at the very long end of the yield curve, thirty-year bonds have been issued approximately every three years throughout our sample, and a single fifty-year bond was issued in 2014 . The contractual characteristics of all 105 bonds and the number of monthly observations for each bond are reported in Appendix A.

All bond prices are represented by their mid-market price as provided by Bloomberg. Following Gürkaynak et al. (2007), securities with less than three months to maturity are excluded from our sample, as the implied yield on these securities often display erratic behavior. $^{4}$

\subsection{Synthetic Zero-Coupon Yields}

The corresponding data for the two-step approach follows the existing literature and represents the universe of bonds by a limited number of synthetic zero-coupon yields. We consider two sources for such synthetic yields. The first data set is produced by the Bank of Canada using the "Merrill Lynch exponential spline model" and is publicly available. ${ }^{5}$ We construct the

\footnotetext{
${ }^{4}$ This may partly reflect a lack of liquidity for these securities or segmented demand for short-term securities by money market funds and other short-term investors.

${ }^{5}$ See Bolder et al. (2004) for a description of the yield curve construction and the algorithm used to filter out "strange" observations. We interpret the elimination of these strange bonds as part of the provided estimation routine. The data set from Bank of Canada can be accessed at the link:
} 


\begin{tabular}{c|c|c|c|c|c}
\hline \hline Maturity & Mean & Mean & Max. & \multicolumn{2}{|c}{ Correlation } \\
\cline { 5 - 6 } in months & diff. & abs. diff. & abs. diff. & Levels & Diff. \\
\hline 3 & 0.78 & 21.52 & 105.24 & 0.982 & 0.410 \\
6 & -1.95 & 11.41 & 65.25 & 0.995 & 0.693 \\
12 & -3.80 & 4.77 & 22.13 & 0.999 & 0.966 \\
24 & -1.13 & 3.22 & 15.85 & 1.000 & 0.986 \\
36 & 1.12 & 2.69 & 11.74 & 1.000 & 0.990 \\
60 & 1.42 & 3.25 & 23.37 & 1.000 & 0.992 \\
84 & -0.71 & 4.85 & 21.57 & 0.999 & 0.989 \\
120 & -5.37 & 5.48 & 19.46 & 1.000 & 0.988 \\
240 & 5.12 & 5.84 & 20.03 & 0.999 & 0.968 \\
360 & -6.63 & 7.86 & 71.43 & 0.995 & 0.848 \\
\hline \hline
\end{tabular}

Table 1: Comparing Two Data Sets of Synthetic Zero-Coupon Yields

The table reports the summary statistics for the mean differences, mean absolute differences, and maximum absolute differences between synthetic Canadian zero-coupon yields from the Bank of Canada and our implementation of the Svensson (1995) curve. These differences are reported in basis points. The last two columns report the correlations between the two yield series for each maturity in levels and first differences, respectively. The data series are monthly covering the period from January 31, 2000, to April 30, 2016.

second data set by estimating the flexible discount function of Svensson (1995) on the same panel of bond prices as used for the one-step approach (see Appendix B for further details). For each data set, we extract synthetic yields with the following ten maturities: $0.25,0.5,1$, $2,3,5,7,10,20$, and 30 years.

Table 1 reports summary statistics for the differences between the two data sets at various maturities. The mean absolute difference for yields in the one- to ten-year maturity range are within 5 basis points and hence small, but larger deviations emerge at the very short and very long maturities. For instance, the mean absolute difference at the six-month and thirty-year maturities are 11 and 8 basis points, respectively, but the largest difference has been 65 basis points for the six-month yield and 71 basis points for the thirty-year yield. The last two columns in Table 1 show the correlations between the two data sets, both when computed in levels and in first-differences. These nonnegligible deviations in the two data sets are also evident, in particular from the correlations in first differences, which differ from one at the short and long maturities.

To further explore these differences, Figure 2 plots the six-month and thirty-year yields from the two data sets. We see notable differences at the six-month maturity at the start of the sample and when the short rate approaches the zero lower bound in 2009. At the thirty-year maturity, the large differences appear mainly at the start of our sample.

Another way to evaluate the magnitude of these differences in synthetic zero-coupon yields http://www.bankofcanada.ca/rates/interest-rates/bond-yield-curves/ 


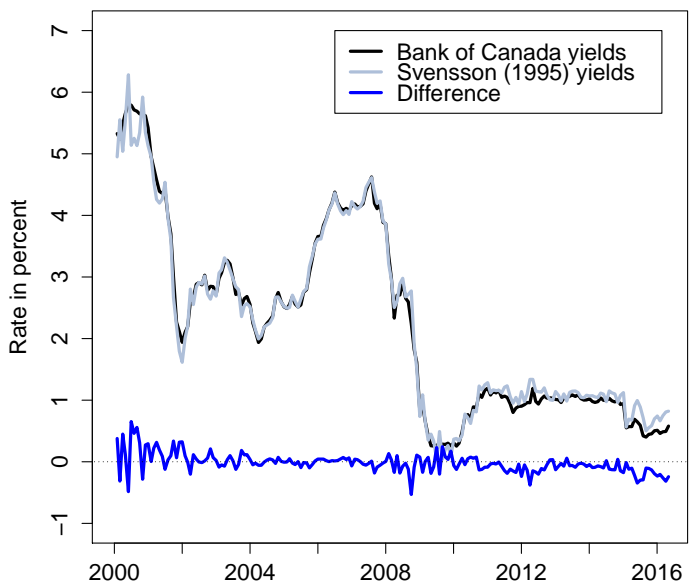

(a) The 6-month yields

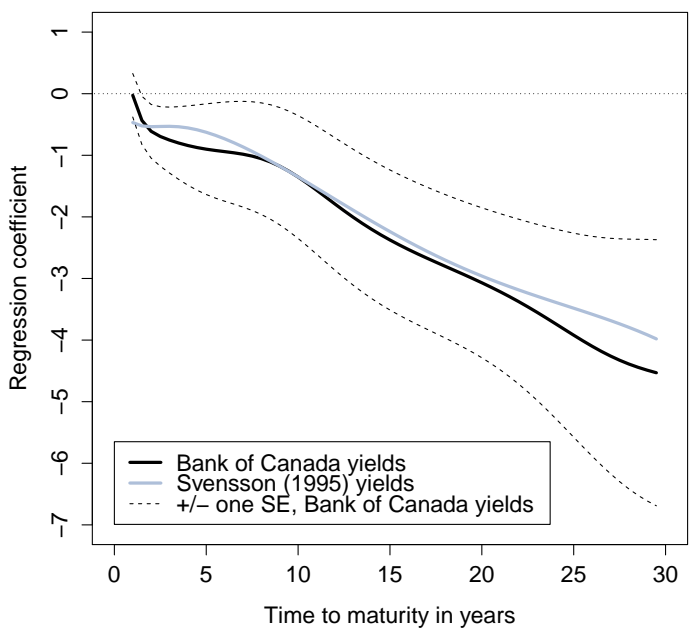

(c) Loadings in Campbell-Shiller regressions

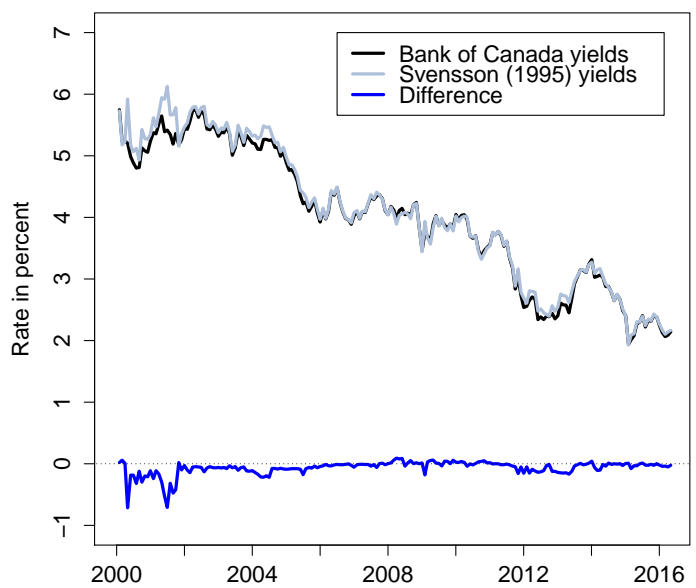

(b) The 30-year yields

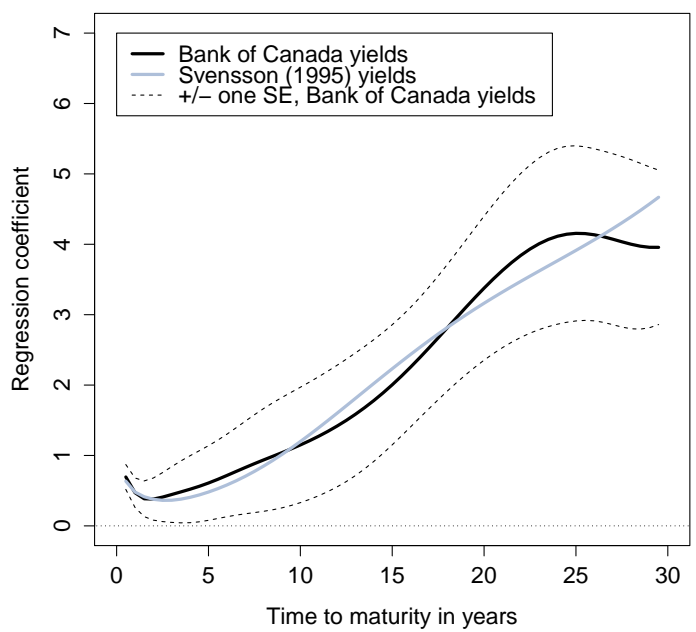

(d) Loadings in forward rate regressions

\section{Figure 2: Two Data Sets of Synthetic Zero-Coupon Yields: Key Differences}

Panel (a) shows the six-month synthetic yields from the Bank of Canada and our implementation of the Svensson (1995) yield curve. Panel (b) shows the thirty-year synthetic yields from the Bank of Canada and our implementation of the Svensson (1995) yield curve. Panel (c) shows $\delta_{k}$ from the regression $y_{t+h}(k-h)-y_{t}(k)=\alpha_{k}+\delta_{k} \frac{h}{k-h}\left(y_{t}(k)-y_{t}(h)\right)+\varepsilon_{t}(k)$ with $h=6$ months, where $y_{t}(k)$ refers to the yield in period $t$ with $k$ months to maturity. Panel (d) shows $\theta(k)$ in the regression $x_{p p r} r_{t+h}(k)=$ $\mu(k)+\theta_{k} x_{t}(k)+\nu_{t+h}(k)$ with $h=6$ months, where $x h p r_{t+h}(k) \equiv h p r_{t+h}(k)-\frac{h}{12} y_{t}(h)$ is the excess holding period return and $h \operatorname{pr}_{t+h}(k) \equiv-\frac{k-h}{12} y_{t+h}(k-h)+\frac{k}{12} y_{t}(k)$ is the holding period return. The variable $x_{t}(k)$ denotes the forward spread $f_{t}^{(k-h, k)}-\frac{h}{12} y_{t}(h)$, where $f_{t}^{(k-h, k)} \equiv \frac{k}{12} y_{t}(k)-\frac{k-h}{12} y_{t}(k-h)$ is the forward rate between time $t+k-h$ and $t+k$.

is to re-visit two classic regressions. The first is due to Campbell and Shiller (1991), where realized returns are regressed on the slope of the yield curve. Panel (c) in Figure 2 shows that 
the loadings in these regressions differ quite a bit at the short and long end of the yield curve but are almost identical in the five- to twenty-year maturity spectrum. The second regression is due to Fama (1976), where realized excess returns are regressed on the slope of the forward curve. Although most regression loadings in Panel (d) coincide closely, we do find substantial differences beyond the twenty-year maturity, as the loadings increase monotonically for the Svensson (1995) yields but not for those provided by the Bank of Canada. Importantly, though, these differences are not statistically significant for both regressions, as the estimated regression loadings based on the Svensson (1995) yields are well within one standard deviation of the estimated coefficients from the Bank of Canada yields.

\section{Empirical Application}

This section presents our empirical application of the one-step approach and compares the results to those obtained from the traditional two-step approach. We proceed by presenting our benchmark DTSM in Section 3.1, while Section 3.2 describes the econometric aspects related to the one- and two-step approach. The estimation results from Canadian bonds are finally discussed in Section 3.3.

\subsection{A Gaussian DTSM}

To capture the factors determining the Canadian yield curve described in the previous section, we focus on the three-factor Gaussian DTSM of Christensen et al. (2011), where the factors represent the familiar level, slope, and curvature of the yield curve. ${ }^{6}$ In this arbitrage-free Nelson-Siegel (AFNS) model, the state vector is denoted by $X_{t}=\left(L_{t}, S_{t}, C_{t}\right)$, where $L_{t}$ is a level factor, $S_{t}$ is a slope factor, and $C_{t}$ is a curvature factor. The instantaneous risk-free rate is defined as

$$
r_{t}=L_{t}+S_{t}
$$

The risk-neutral (or $Q-$ ) dynamics of the state variables are given by the stochastic differential equations

$$
\left(\begin{array}{c}
d L_{t} \\
d S_{t} \\
d C_{t}
\end{array}\right)=\left(\begin{array}{ccc}
0 & 0 & 0 \\
0 & -\lambda & \lambda \\
0 & 0 & -\lambda
\end{array}\right)\left(\begin{array}{c}
L_{t} \\
S_{t} \\
C_{t}
\end{array}\right) d t+\Sigma\left(\begin{array}{c}
d W_{t}^{L, Q} \\
d W_{t}^{S, Q} \\
d W_{t}^{C, Q}
\end{array}\right)
$$

Here, $d W^{i, Q}$ for $i=\{L, S, C\}$ denotes independent Wiener processes and $\Sigma$ is a constant covariance matrix with dimensions $3 \times 3 .^{7}$ As shown in Christensen et al. (2011), this implies

\footnotetext{
${ }^{6}$ Although the model is not formulated using the canonical form of affine DTSMs in Dai and Singleton (2000), it can be viewed as a restricted version of this model class.

${ }^{7}$ As discussed in Christensen et al. (2011), the unit root in the level factor implies that the model is only free of arbitrage for bonds with a finite horizon. For our sample of Canadian bonds described in Section 2,
} 
that the zero-coupon bond yield at maturity $\tau$ is given by

$$
y\left(\tau ; X_{t}\right)=L_{t}+\left(\frac{1-e^{-\lambda \tau}}{\lambda \tau}\right) S_{t}+\left(\frac{1-e^{-\lambda \tau}}{\lambda \tau}-e^{-\lambda \tau}\right) C_{t}-\frac{A(\tau)}{\tau},
$$

where $A(\tau)$ is a convexity term that 'adjusts' the functional form in Nelson and Siegel (1987) to ensure absence of arbitrage. ${ }^{8}$

The model is closed by adopting the essentially affine specification for the market price of risk $\Gamma_{t}$ as in Duffee (2002). That is, we let $\Gamma_{t}=\gamma^{0}+\gamma^{1} X_{t}$, where $\gamma^{0} \in \mathbf{R}^{3}$ and $\gamma^{1} \in \mathbf{R}^{3 \times 3}$ contain unrestricted parameters. The physical (or $P_{-}$) dynamics of the three factors in the AFNS model are therefore given by

$$
\left(\begin{array}{c}
d L_{t} \\
d S_{t} \\
d C_{t}
\end{array}\right)=\left(\begin{array}{ccc}
\kappa_{11}^{P} & \kappa_{12}^{P} & \kappa_{13}^{P} \\
\kappa_{21}^{P} & \kappa_{22}^{P} & \kappa_{23}^{P} \\
\kappa_{31}^{P} & \kappa_{32}^{P} & \kappa_{33}^{P}
\end{array}\right)\left(\left(\begin{array}{c}
\theta_{1}^{P} \\
\theta_{2}^{P} \\
\theta_{3}^{P}
\end{array}\right)-\left(\begin{array}{c}
L_{t} \\
S_{t} \\
C_{t}
\end{array}\right)\right) d t+\Sigma\left(\begin{array}{c}
d W_{t}^{L, P} \\
d W_{t}^{S, P} \\
d W_{t}^{C, P}
\end{array}\right)
$$

where $\kappa_{i, j}^{P}$ and $\theta_{i}^{P}$ are free parameters, subject to $X_{t}$ being stationary under the $P$-measure.

\subsection{Estimation Methodology in the One-Step and Two-Step Approach}

To describe the econometric implementation of the one-step approach, let $P_{t}^{i}(\tau, C)$ denote the price at time $t$ of the $i$ th coupon bond, which matures at time $t+\tau$ and pays the coupon $C$ semi-annually. In the absence of arbitrage, the price of this coupon bond must equal the discounted sum of all remaining payments, i.e.,

$$
P_{t}^{i}(\tau, C)=\frac{C}{2} \frac{\left(t_{1}-t\right)}{1 / 2} P_{t}^{\mathrm{zc}}\left(t_{1}-t\right)+\sum_{j=2}^{N} \frac{C}{2} P_{t}^{\mathrm{zc}}\left(t_{j}-t\right)+P_{t}^{\mathrm{zc}}\left(t_{N}-t\right)
$$

where $t<t_{1}<\ldots<t_{N}=\tau$. Here, $P_{t}^{\mathrm{zc}}(\tau)=\exp \left\{-y\left(\tau ; X_{t}\right) \tau\right\}$ denotes the price of the zerocoupon bond with $\tau$ years to maturity, and $y\left(\tau ; X_{t}\right)$ is the zero-coupon yield from the DTSM. The corresponding bond price in the data is denoted $P_{t}^{i, D a t a}(\tau, C)$. To ensure that the errors of the DTSM are comparable across bonds with different maturities, we scale each bond price by its duration. Here, we apply the model-free measure of Macaulay, which is calculated before the model estimation and denoted $D_{t}^{i, D a t a}(\tau, C)$. The measurement equation for the $i$ th bond price in the one-step approach is therefore given by

$$
\frac{P_{t}^{i, \text { Data }}(\tau, C)}{D_{t}^{i, \text { Data }}(\tau, C)}=\frac{P_{t}^{i}(\tau, C)}{D_{t}^{i, \text { Data }}(\tau, C)}+\varepsilon_{t}^{i},
$$

and most other sovereign bond markets, this restriction is not binding and therefore of no practical relevance.

${ }^{8}$ The analytical expression for the yield-adjustment term $A(\tau)$ is provided in Christensen et al. (2011). 
where $\varepsilon_{t}^{i}$ represents independent and Gaussian distributed measurement errors with mean zero and a common standard deviation $\sigma_{\varepsilon}$, i.e., $\varepsilon_{t}^{i} \sim \mathcal{N} \mathcal{I D}\left(0, \sigma_{\varepsilon}^{2}\right) .{ }^{9}$ The state transition dynamics for $X_{t}$ under the $P$-measure is given by equation (4).

As is commonly assumed, the state variables are taken to be unobserved and must be estimated along with the model parameters $\psi$ from the panel of bond prices. The nonlinear relationship between the states $X_{t}$ and the price of a coupon bond $P_{t}^{i}(\tau, C)$ in equation (5) implies that the AFNS model cannot be estimated with the standard Kalman filter. Instead, we use the well-known extended Kalman filter (EKF) to obtain an approximated log-likelihood function $L^{E K F}(\psi)$, which serves as the basis for estimating $\psi$ by quasi-maximum likelihood (QML), as described in further detail in Appendix C.

The econometric implementation of the two-step approach is well-known but summarized here for completeness. Let the synthetic zero-coupon yields in the data be denoted by $y_{t}^{D a t a}(\tau)$, and let $y\left(\tau, X_{t}\right)$ denote the corresponding yield from the DTSM. The measurement equation is then given by

$$
y_{t}^{\text {Data }}(\tau)=y\left(\tau, X_{t}\right)+\varepsilon_{t}(\tau),
$$

for a selection of constant maturities as indexed by $\tau$. The variable $\varepsilon_{t}(\tau) \sim \mathcal{N} \mathcal{I D}\left(0, \sigma_{\varepsilon}^{2}\right)$ and accounts for estimation errors in the construction of these synthetic zero-coupon yields within the first step. The state transition dynamics for $X_{t}$ under the $P$-measure is similar to the one-step approach and given by equation (4). For the AFNS model, the zero-coupon yields are affine in $X_{t}$ as seen from equation (3), and all model parameters $\psi$ are therefore estimated by maximum likelihood based on the Kalman filter.

\subsection{Estimation Results for the AFNS Model}

The estimated model parameters in the AFNS model are reported in Table 2 when using the one-step and the two-step approach. The conventional two-step approach is implemented on the two samples of synthetic yields discussed in Section 2.2 to explore whether the highlighted differences in the two data sets affect the estimated model parameters. Hence, the one-step approach uses all available bond prices with maturities exceeding three months, whereas the two-step approach only uses the ten maturities selected in Table 1. In the interest of simplicity, we focus on the most parsimonious version of the AFNS model with independent factor dynamics in this section. This restriction comes at practically no loss of generality for the reported results, as the estimated factors and model fit are insensitive to omitting the off-diagonal terms in $\mathcal{K}^{P}$ and $\Sigma .{ }^{10}$

\footnotetext{
${ }^{9} \mathrm{As}$ is common, we also assume that these errors are uncorrelated to the state innovations in equation (4), and hence to the factors in $X_{t}$ at all leads and lags.

${ }^{10}$ See for instance Christensen et al. (2011), who also show that this restricted model often does better at forecasting yields out of sample than the most flexible version of the AFNS model, where $\mathcal{K}^{P}$ and $\Sigma$ are unrestricted.
} 


\begin{tabular}{|c|c|c|c|c|c|c|}
\hline \multirow{3}{*}{ Par. } & \multirow{2}{*}{\multicolumn{2}{|c|}{ One-step approach }} & \multicolumn{4}{|c|}{ Two-step approach } \\
\hline & & & \multicolumn{2}{|c|}{ Bank of Canada yields } & \multicolumn{2}{|c|}{ Svensson (1995) yields } \\
\hline & Est & $\mathrm{SE}$ & Est & $\mathrm{SE}$ & Est & $\mathrm{SE}$ \\
\hline$\kappa_{11}^{P}$ & 0.1060 & 0.0763 & 0.2172 & 0.3086 & 0.0835 & 0.1327 \\
\hline$\kappa_{22}^{P}$ & 0.2157 & 0.1443 & 0.1839 & 0.1696 & 0.2982 & 0.1969 \\
\hline$\kappa_{33}^{P}$ & 0.7255 & 0.3649 & 0.4214 & 0.2675 & 0.3543 & 0.2301 \\
\hline$\sigma_{11}$ & 0.0052 & 0.0001 & 0.0071 & 0.0001 & 0.0052 & 0.0001 \\
\hline$\sigma_{22}$ & 0.0103 & 0.0010 & 0.0085 & 0.0005 & 0.0103 & 0.0004 \\
\hline$\sigma_{33}$ & 0.0207 & 0.0015 & 0.0197 & 0.0013 & 0.0212 & 0.0013 \\
\hline$\theta_{1}^{P}$ & 0.0529 & 0.0034 & 0.0542 & 0.0111 & 0.0477 & 0.0143 \\
\hline$\theta_{2}^{\stackrel{P}{P}}$ & -0.0275 & 0.0093 & -0.0295 & 0.0136 & -0.0251 & 0.0088 \\
\hline$\theta_{3}^{P}$ & -0.0230 & 0.0060 & -0.0187 & 0.0129 & -0.0181 & 0.0156 \\
\hline$\lambda$ & 0.3747 & 0.0105 & 0.3070 & 0.0047 & 0.4511 & 0.0051 \\
\hline
\end{tabular}

Table 2: Parameter Estimates in the AFNS Model

This table reports the estimated parameters (Est) in the AFNS model with independent factors and their standard errors (SE) using either the one-step or the two-step approach. The SE in the one-step approach are computed by pre- and post-multiplying the variance of the score by the inverse of the Hessian matrix, as outlined in Harvey (1989). The SE in the two-step approach are computed from the inverse of the variance of the score. The data are monthly and cover the period from January 31 , 2000, to April 29, 2016.

We first note that all elements in $\mathcal{K}^{P}$ and $\theta$ are estimated very inaccurately in the three data sets, which is a well-known characteristic of estimating persistent autoregressive processes over a relatively short time span. The diagonal elements in $\Sigma$ and $\lambda$ are estimated much more accurately and reveal some notable differences. First, the volatility of the level factor $\sigma_{11}$ is 0.0071 in the two-step approach based on yields from Bank of Canada, but only 0.0052 in the one-step approach and in the two-step approach based on Svensson (1995) yields. Second, the volatility of the slope factor $\sigma_{22}$ is 0.0085 in the two-step approach using yields from the Bank of Canada, whereas we find $\sigma_{22}=0.0103$ in the two other data sets. Finally, the Nelson-Siegel parameter $\lambda$ is 0.375 in the one-step approach, 0.305 in the two-step approach based on Bank of Canada yields, and 0.451 in the two-step approach based on Svensson (1995) yields. These findings reveal that the estimated parameters in a DTSM are affected by using synthetic zero-coupon yields as opposed to the underlying market prices on coupon bonds, and that even small differences between synthetic yields of the same maturity can matter for the estimation results.

Figure 3 shows the filtered states from estimating the AFNS model. Each of the states are highly correlated across the three data sets as expected, but we also observe some differences. For instance, the level factor in the two-step approach based on Bank of Canada yields is generally 30 to 40 basis points above the estimated level factor from the one-step approach, whereas, for the slope factor, we generally find the opposite ordering between the two data sets. 


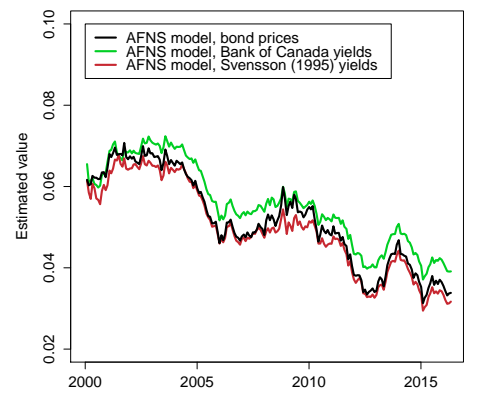

(a) $L_{t}$

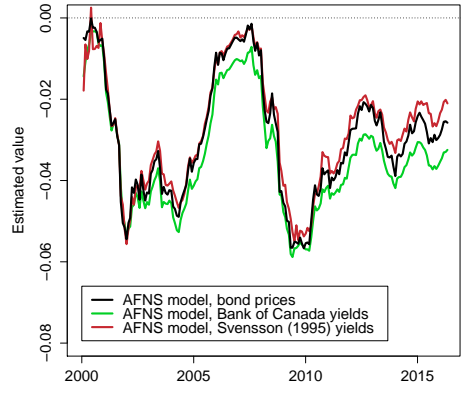

(b) $S_{t}$

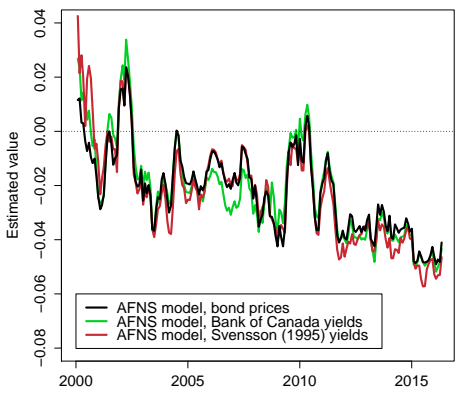

(c) $C_{t}$

\section{Figure 3: Estimated States in the AFNS Model}

Illustration of the estimated level, slope, and curvature factors in the AFNS model with independent factor dynamics. The data are monthly and cover the period from January 31, 2000, to April $29,2016$.

Somewhat smaller differences appear in the state estimates between the one-step approach and the two-step approach based on Svensson (1995) yields, although the two estimates of the curvature factor behave differently at the start and at the end of the sample.

Table 3 evaluates the ability of the AFNS model to match market prices on coupon bonds. The pricing errors are here computed based on the implied yield on each coupon bond to make these errors comparable across securities. That is, for the price on the $i$ th coupon bond $P_{t}^{i}(\tau, C)$, we find the value of $y_{t}^{i, c}$ that solves

$$
P_{t}^{i}(\tau, C)=\frac{C}{2} \frac{\left(t_{1}-t\right)}{1 / 2} \exp \left\{-y_{t}^{i, c}\left(t_{1}-t\right)\right\}+\sum_{j=2}^{N} \frac{C}{2} \exp \left\{-y_{t}^{i, c}\left(t_{j}-t\right)\right\}+\exp \left\{-y_{t}^{i, c}\left(t_{N}-t\right)\right\} .
$$

For the model-implied estimate of this bond price, denoted $\hat{P}_{t}^{i}(\tau, C)$, we find the corresponding implied yield $\hat{y}_{t}^{i, c}$ and report the pricing error as $y_{t}^{i, c}-\hat{y}_{t}^{i, c} .{ }^{11}$ Table 3 shows that the two-step approach provides a fairly tight fit to the underlying coupon bond prices with an overall root mean squared error (RMSE) of 8.31 basis points for the Bank of Canada yields and 7.90 basis points for the Svensson (1995) yields. We emphasize that both the states and the model estimates in the AFNS model are here obtained from syntehtic zero-coupon yields. Thus, the conventional two-step approach provides a fairly accurate fit to the underlying coupon bonds, although these bonds only enter indirectly through the synthetic zero-coupon yields in the estimation of the AFNS model. Another and equally important observation is that the one-step approach delivers an even better fit to these coupon bonds with an overall RMSE of only 5.79 basis points. Compared to the overall RMSE in the two-step approach, this

\footnotetext{
${ }^{11}$ Scaling bond prices by duration in equation (6) when estimating DTSMs in the one-step approach serves as a first-order approximation to the implied yield on a coupon bond. We prefer scaling bond prices by duration when estimating DTSMs in the one-step approach, because it is computationally much less demanding than estimating DTSMs based on the fixed-point problem in equation (7).
} 


\begin{tabular}{c|c|cc|cc|ccc|c}
\hline \hline \multirow{2}{*}{$\begin{array}{c}\text { Maturity } \\
\text { bucket }\end{array}$} & \multirow{2}{*}{$\begin{array}{l}\text { No. } \\
\text { obs. }\end{array}$} & \multicolumn{2}{|c|}{ One-step approach } & \multicolumn{5}{|c|}{ Two-step approach } & \multicolumn{2}{c}{$\begin{array}{c}\text { Svensson (1995) } \\
\text { zero-coupon yields }\end{array}$} \\
\cline { 3 - 8 } & & Mean & RMSE & Mean & RMSE & Mean & RMSE & Mean & RMSE \\
\hline $0-2$ & 1,472 & -0.10 & 5.74 & 2.40 & 7.87 & 0.33 & 9.81 & -1.08 & 8.83 \\
$2-4$ & 1,098 & 0.44 & 4.74 & 1.78 & 7.20 & 2.02 & 5.61 & 0.87 & 4.27 \\
$4-6$ & 744 & -0.39 & 3.85 & 1.04 & 4.37 & -1.23 & 4.61 & 0.40 & 3.50 \\
$6-8$ & 404 & -1.24 & 5.47 & 0.04 & 5.02 & -3.12 & 6.61 & -1.89 & 4.50 \\
$8-10$ & 477 & -2.54 & 6.07 & -2.27 & 6.61 & -4.62 & 7.81 & -2.95 & 5.43 \\
$10-12$ & 289 & -1.14 & 6.20 & -1.09 & 8.56 & -2.01 & 8.35 & -2.06 & 5.84 \\
$12-14$ & 155 & 3.79 & 6.72 & 4.70 & 11.98 & 4.79 & 11.18 & 2.01 & 3.65 \\
$14-16$ & 168 & 0.77 & 4.32 & -1.61 & 9.05 & 0.36 & 8.28 & 0.35 & 2.87 \\
$16-18$ & 179 & 0.71 & 4.66 & -1.97 & 9.89 & 0.94 & 8.84 & 0.24 & 3.80 \\
$18-20$ & 192 & 1.71 & 4.33 & 2.02 & 8.89 & 4.88 & 8.64 & 0.68 & 3.60 \\
$20-22$ & 186 & 3.45 & 4.97 & 5.06 & 10.06 & 7.36 & 10.32 & 2.32 & 4.58 \\
$22-24$ & 142 & 0.60 & 4.47 & 2.37 & 7.27 & 4.62 & 7.09 & 1.39 & 3.59 \\
$24-26$ & 124 & -0.08 & 5.01 & 3.75 & 8.37 & 4.67 & 7.43 & 1.63 & 3.56 \\
$26-28$ & 113 & -5.58 & 8.36 & 0.73 & 5.90 & 0.33 & 4.49 & -1.32 & 3.33 \\
$28<$ & 288 & -5.01 & 11.91 & 6.36 & 18.13 & 0.88 & 8.69 & -2.75 \\
\hline All bonds & 6,031 & -0.36 & 5.79 & 1.50 & 8.31 & 0.42 & 7.90 & -0.44 & 5.37 \\
\hline \hline
\end{tabular}

Table 3: Summary Statistics of Bond Fitted Errors in the AFNS Model

This table reports the mean pricing errors (Mean) and the root mean-squared pricing errors (RMSE) of the Canadian bond prices for the AFNS model with independent factors estimated on three different data sets: (1) the universe of Canadian coupon bond prices, (2) zero-coupon yields constructed by the Bank of Canada, and (3) zero-coupon yields constructed from Canadian coupon bond prices using the Svensson (1995) yield curve. The final two columns report the corresponding statistics for the constructed Svensson (1995) yield curve. The pricing errors are reported in basis points and computed as the difference between the implied yield on the coupon bond and the model-implied yield on this bond. The data are monthly and cover the period from January 31, 2000, to April 29, 2016.

corresponds to an $44 \%$ and $36 \%$ improvement when using the Bank of Canada yields and the Svensson (1995) yields, respectively. This shows that the first step in the conventional two-step approach may add a considerable amount of noise to the predicted bond prices from the estimated DTSM.

In the final two columns of Table 3, we benchmark these results from the AFNS model to the fit of the Svensson (1995) discount function, that is, we compute the predicted price of a given coupon bond from the synthetic Svensson (1995) yields, which we then convert into the implied yield using equation (7) to obtain the pricing error. As expected, the RMSEs for bonds with maturities exceeding two years are all smaller for the Svensson (1995) discount function when compared to any of the estimated versions of the AFNS model. However, the deterioration in fit for the estimated AFNS model based on the one-step approach is surprisingly small except for very long-term bonds with more than 26 years to maturity. Even more surprising are the results for bonds within the zero to two-year maturity bucket, where the estimated AFNS model based on the one-step approach has a RMSE of only 5.74 basis points and hence does better than the Svensson (1995) discount function with a RMSE of 8.83 basis points. When accounting for the large number of bonds in this maturity bucket, 
we find that the overall RMSE of the Svensson (1995) discount function is 5.78 basis points and hence basically identical to that of the AFNS model from the one-step approach with an overall RMSE of 5.79 basis points.

\section{Extensions}

The present section explores whether we can improve the ability of the AFNS model to fit coupon bonds in the one-step approach. Section 4.1 replaces the proposed QML estimator in the one-step approach with a fully efficient maximum likelihood estimator. Section 4.2 extends the AFNS model with a shadow-rate specification to accommodate the zero lower bond, while the effects of extending the AFNS model with two additional factors to better fit long-term bonds are explored in Section 4.3. From a methodological perspective, these extensions illustrate that the proposed one-step approach is applicable to i) fully efficient maximum likelihood estimation, ii) nonlinear DTSMs, and iii) models with more than three factors. As in the previous section, we benchmark the performance of the one-step approach to those from the conventional two-step approach based on synthetic zero-coupon yields from the Bank of Canada and the Svensson (1995) discount function.

\subsection{Maximum Likelihood Estimation in the One-Step Approach}

It is well-known that the adopted QML estimator in the one-step approach based on the EKF induces an efficiency loss compared to maximum likelihood (ML), but it is perhaps less recognized that consistency of this QML estimator cannot be established as the sample size $T$ tends to infinity. ${ }^{12}$ To explore whether the performance of the one-step approach can be improved by adopting a better estimator, we next show how the one-step approach can be implemented with a fully efficient ML estimator.

We have so far adopted a Bayesian perspective when filtering out the states in both the one-step and two-step approach. But the one-step approach is characterized by a large set of observables in the cross-sectional dimension, and it therefore seems natural to adopt a classical perspective to filtering, as commonly considered in the estimation of large factor models (see, for instance, Bai and $\mathrm{Ng}$ (2002) and Bai (2003)). ${ }^{13}$ That is, we now consider the states $X_{1: T} \equiv\left\{X_{t}^{\prime}\right\}_{t=1}^{T}$ as parameters along with the model parameters $\psi \cdot{ }^{14}$ The main advantage

\footnotetext{
${ }^{12}$ This is because the approximated nature of the EKF implies that the conditional first and second moments for the prediction errors related to coupon bond prices cannot be computed exactly at the true model parameters, see Bollerslev and Wooldridge (1992) and Andreasen (2013).

${ }^{13}$ A classical perspective to filtering has also recently been considered by Andreasen and Christensen (2015) when estimating DTSMs and by Andersen et al. (2015) when estimating option pricing models.

${ }^{14}$ The curve-fitting procedure of Svensson (1995), Bliss (1996), and Gürkaynak et al. (2007, 2010) among others adopt the same classical perspective, as they estimate a parametric model for a daily yield curve, where the "states" in these curves are treated as parameters and estimated from a large panel of bond prices.
} 
of considering the states $X_{1: T}$ as parameters is that the likelihood function can be evaluated without simulation for a nonlinear DTSM with Gaussian innovations and measurement errors, and this in turn makes full ML estimation feasible within the one-step approach.

To realize this, let $\tilde{\psi} \equiv\left[\begin{array}{ll}\psi & X_{1: T}\end{array}\right]$ denote the extended set of model parameters and let $n_{y, t}$ denote the number of bond prices in period $t$, which we collect in $Y_{t}$. Hence, $Y_{1: T} \equiv\left\{Y_{t}\right\}_{t=1}^{T}$ refers to the entire sample of bond prices. The relation between bond prices and the states is then expressed condensely by the measurement equation

$$
Y_{t}=g\left(X_{t} ; \psi\right)+\varepsilon_{t}
$$

where $g\left(X_{t} ; \psi\right)$ is a nonlinear function in $X_{t}$ and $\varepsilon_{t} \sim \mathcal{N} \mathcal{I D}\left(0, R_{\varepsilon, t}\right) .{ }^{15}$ The state transition dynamics under the $P$-measure is after an appropriate Euler-discretization given by

$$
X_{t+1}=h\left(X_{t} ; \psi\right)+w_{t+1},
$$

where $h\left(X_{t} ; \psi\right)$ is a potentially nonlinear function in $X_{t}$ and $w_{t+1} \sim \mathcal{N} \mathcal{I D}\left(0, R_{w}\right)$. Given the imposed distributional assumptions on the system in equations (8) and (9), the log-likelihood function $L\left(\tilde{\psi} \mid Y_{1: T}\right)$ is then proportional to (see Durbin and Koopman (2001))

$$
\begin{aligned}
L\left(\tilde{\psi} \mid Y_{1: T}\right) \propto & \frac{T}{2} \log \left|R_{w}^{-1}\right|-\frac{1}{2} \sum_{t=1}^{T}\left(X_{t+1}-h\left(X_{t} ; \psi\right)\right)^{\prime} R_{w}^{-1}\left(X_{t+1}-h\left(X_{t} ; \psi\right)\right) \\
& +\sum_{t=1}^{T} \frac{1}{2} \log \left|R_{\varepsilon, t}^{-1}\right|-\frac{1}{2} \sum_{t=1}^{T}\left(Y_{t}-g\left(X_{t} ; \psi\right)\right)^{\prime} R_{\varepsilon, t}^{-1}\left(Y_{t}-g\left(X_{t} ; \psi\right)\right) .
\end{aligned}
$$

The ML estimator is then given by

$$
\widehat{\tilde{\psi}}_{M L}=\underset{\tilde{\psi} \in \tilde{\Psi}}{\arg \max } L\left(\tilde{\psi} \mid Y_{1: T}\right)
$$

where $\tilde{\Psi}$ denotes the feasible set for $\tilde{\psi}$. To make this optimization problem computationally feasible, we use the procedure in Durbin and Koopman (2001) to numerically concentrate out $X_{1: T}$ from $L\left(\tilde{\psi} \mid Y_{1: T}\right)$ for a given value of $\psi$. As explained in Appendix $\mathrm{D}$, this is done by iterating the Kalman filter and smoother on a linearized version of the system in equations (8) and (9), where convergence for the AFNS model typically is achieved within five iterations. ${ }^{16}$ The asymptotic distribution of $\hat{\psi}_{M L}$ when $n_{y, t} \longrightarrow \infty$ for all $t$ and $T \longrightarrow \infty$ at the same rate

\footnotetext{
${ }^{15}$ The subscript $t$ on $R_{\varepsilon, t}$ indicates that its dimension adapts to the available number of bonds throughout the sample.

${ }^{16}$ The specification in (9) omits nonlinearities between the states and the innovations, but this is without loss of generality, as shown in Appendix E. Hence, the proposed ML estimator may also be applied to DTSMs with stochastic volatility.
} 


\begin{tabular}{c|cc|cc}
\hline \hline \multirow{2}{*}{ Par. } & \multicolumn{2}{|c|}{ QML } & \multicolumn{2}{c}{ ML } \\
\cline { 2 - 5 } & Est & SE & Est & SE \\
\hline$\kappa_{11}^{P}$ & 0.1060 & 0.0763 & 0.0720 & 0.1353 \\
$\kappa_{22}^{P}$ & 0.2157 & 0.1443 & 0.2038 & 0.1846 \\
$\kappa_{33}^{P}$ & 0.7255 & 0.3649 & 0.3748 & 0.3177 \\
\hline$\sigma_{11}$ & 0.0052 & 0.0001 & 0.0052 & 0.0000 \\
$\sigma_{22}$ & 0.0103 & 0.0010 & 0.0095 & 0.0003 \\
$\sigma_{33}$ & 0.0207 & 0.0015 & 0.0170 & 0.0010 \\
\hline$\theta_{1}^{P}$ & 0.0529 & 0.0034 & 0.0497 & 0.0158 \\
$\theta_{2}^{P}$ & -0.0275 & 0.0093 & -0.0247 & 0.0133 \\
$\theta_{3}^{P}$ & -0.0230 & 0.0060 & -0.0188 & 0.0140 \\
\hline$\lambda$ & 0.3747 & 0.0105 & 0.3774 & 0.0015 \\
\hline \hline
\end{tabular}

Table 4: ML Estimates of the AFNS Model

This table reports the estimated parameters (Est) in the AFNS model with independent factors in the one-step approach, using either QML or ML. The standard errors (SE) for the QML are computed by pre- and post-multiplying the variance of the score by the inverse of the Hessian matrix, as outlined in Harvey (1989). The SE for the ML estimates are obtained as the inverse of the variance for the concentrated score function. The data are monthly and cover the period from January 31, 2000, to April 29, 2016.

as $n_{y, t}$ is multivariate normal, and the standard errors are given by the inverse of the variance of the score for the concentrated log-likelihood function (see Hahn and Newey (2004)). ${ }^{17}$

The ML estimates are provided in Table 4. For the AFNS model we find very small differences between the ML and the QML estimates. In particular, the two estimates of $\lambda$ are almost identical. Hence, estimating the AFNS model by ML in the one-step approach does not improve the ability of the model to fit coupon bonds compared to those reported in Table 3 based on the QML estimator. ${ }^{18}$ In the remaining part of the paper, we therefore only report results using the QML estimator, which is computationally somewhat faster than the ML estimation described above.

\subsection{A Shadow-Rate Model}

Given the very low policy rates in many economies during the recent financial crisis, it has become popular to account for the zero lower bound (ZLB) in DTSMs. Although short rates are close to zero for only a limited period in our Canadian sample (as seen from Figure $2(\mathrm{a})$ ), it is still possible that the ZLB may affect the shape and dynamics of the yield curve

\footnotetext{
${ }^{17}$ It is well-known from the literature on fixed-effects in panel models that $\hat{\psi}_{M L}$ may be affected by the incidental bias $B^{i n c} / n_{y}$, which in our case arises from the uncertainty attached to estimating an increasing number of states $X_{1: T}$ as $T$ grows. However, the states are estimated very accurately in multi-factor DTSMsas shown in Section 5 below - and the incidental bias is therefore unlikely to be important for estimating DTSMs with a reasonable number of cross-sectional observations $n_{y}$. An analytical expression for the incidental bias $B^{i n c}$ may be derived following the procedure in Hahn and Newey (2004).

${ }^{18}$ The corresponding version of Table 3 based on the ML estimates are available upon request.
} 


\begin{tabular}{|c|c|c|c|c|c|c|}
\hline \multirow{3}{*}{ Par. } & \multirow{2}{*}{\multicolumn{2}{|c|}{ One-step approach }} & \multicolumn{4}{|c|}{$\begin{array}{c}\text { Two-step approach } \\
\end{array}$} \\
\hline & & & \multicolumn{2}{|c|}{ Bank of Canada yields } & \multicolumn{2}{|c|}{ Svensson (1995) yields } \\
\hline & Est & $\mathrm{SE}$ & Est & $\mathrm{SE}$ & Est & SE \\
\hline$\kappa_{11}^{P}$ & 0.1450 & 0.0891 & 0.1373 & 0.0669 & 0.0521 & 0.0468 \\
\hline$\kappa_{22}^{P}$ & 0.1066 & 0.0819 & 0.1074 & 0.0701 & 0.5166 & 0.1828 \\
\hline$\kappa_{33}^{P}$ & 0.4337 & 0.2897 & 0.3503 & 0.3573 & 0.2646 & 0.3935 \\
\hline$\sigma_{11}$ & 0.0073 & 0.0002 & 0.0083 & 0.0003 & 0.0059 & 0.0002 \\
\hline$\sigma_{22}$ & 0.0122 & 0.0011 & 0.0098 & 0.0008 & 0.0114 & 0.0011 \\
\hline$\sigma_{33}$ & 0.0180 & 0.0021 & 0.0205 & 0.0021 & 0.0220 & 0.0026 \\
\hline$\theta_{1}^{P}$ & 0.0546 & 0.0034 & 0.0546 & 0.0115 & 0.0555 & 0.0242 \\
\hline$\theta_{2}^{P}$ & -0.0396 & 0.0087 & -0.0248 & 0.0135 & -0.0231 & 0.0046 \\
\hline$\theta_{3}^{P}$ & -0.0248 & 0.0117 & -0.0250 & 0.0135 & -0.0238 & 0.0219 \\
\hline$\lambda$ & 0.3920 & 0.0123 & 0.3473 & 0.0135 & 0.4754 & 0.0149 \\
\hline
\end{tabular}

Table 5: Estimated Parameters in the B-AFNS Model

This table reports the estimated parameters (Est) in the B-AFNS model with independent factors and their standard errors (SE) using either the one-step or the two-step approach. The SE are in all cases computed by pre- and post-multiplying the variance of the score by the inverse of the Hessian matrix, as outlined in Harvey (1989). The data are monthly and cover the period from January 31, 2000, to April 29, 2016.

even during episodes of near-zero interest rates (see, e.g., Swanson and Williams (2014)). To enforce the ZLB in the AFNS model, we follow Black (1995) and introduce the shadow rate $s_{t}=L_{t}+S_{t}$ and let $r_{t}=\max \left\{0, s_{t}\right\}$, as in Christensen and Rudebusch (2015). All other aspects of this B-AFNS model remain as described above for the AFNS model. ${ }^{19}$ The expression for zero-coupon yields in the B-AFNS model is not available in closed form but approximated numerically using the accurate method of Krippner (2013). ${ }^{20}$

Table 5 shows that all elements in $\mathcal{K}^{P}$ and $\theta^{P}$ in the B-AFNS model are also estimated very inaccurately across the three data sets. The volatility parameters in $\Sigma$ are estimated much more precisely and are generally higher in the B-AFNS model when compared to the AFNS model. Figure 4 shows that this difference is mainly explained by greater factor variability after 2008, because the shadow-rate specification in the B-AFNS model allows the factors to move more freely than seen in the AFNS model without violating the ZLB. We also find that $\lambda$ is estimated to be somewhat higher in all three data sets when accounting for the ZLB. Similar to the pattern observed for the AFNS model, the estimate of $\lambda$ in the B-AFNS model within the one-step approach lies in between those from the two-step approach, as $\lambda$ is 0.392 in the one-step approach, 0.347 in the two-step approach based on Bank of Canada yields, and 0.475 in the two-step approach using Svensson (1995) yields.

\footnotetext{
${ }^{19}$ Following Kim and Singleton (2012), the prefix "B-" refers to a shadow-rate model in the spirit of Black (1995).

${ }^{20}$ See also Christensen and Rudebusch $(2015,2016)$ for further details on this approximation and its accuracy.
} 


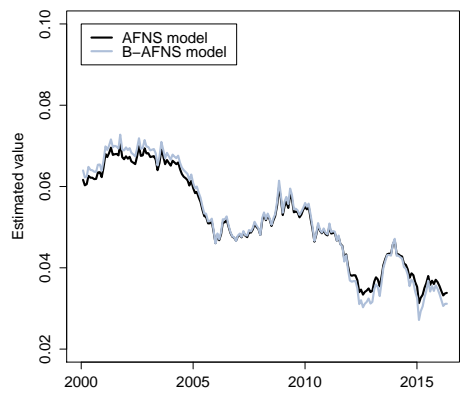

(a) $L_{t}$

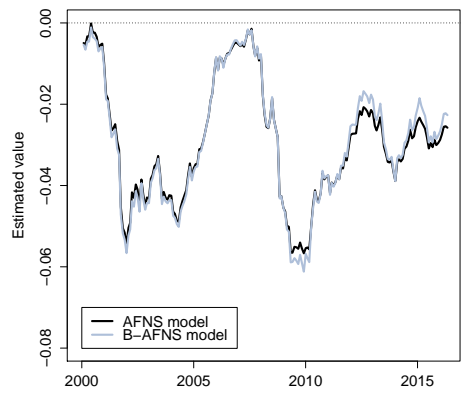

(b) $S_{t}$

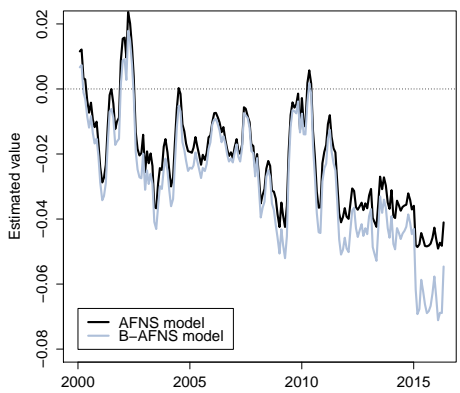

(c) $C_{t}$

Figure 4: Estimated States in the One-Step Approach

This figure reports the filtered estimates of level, slope, and curvature in the AFNS and B-AFNS model. The data are monthly and cover the period from January 31, 2000, to April 29, 2016.

Table 6 reports the pricing errors of the B-AFNS model for the underlying coupon bonds. For the one-step approach and both versions of the two-step approach, we find slightly smaller RMSEs in the B-AFNS model compared to the AFNS model. For instance, the overall RMSE falls by $3 \%$ from 5.79 to 5.62 basis points in the one-step approach. Thus, accounting for the ZLB does not materially improve the ability of the AFNS model to match Canadian coupon bond prices.

\subsection{A Five-Factor Model}

The main motivation of Gürkaynak et al. (2007) to prefer the Svensson (1995) curve over the simpler specification of Nelson and Siegel (1987) is that the Svensson (1995) curve allows for an additional 'hump' that helps fit U.S. bond yields beyond the ten- to fifteen-year maturity spectrum. ${ }^{21}$ The AFNS model may potentially also benefit from additional dynamics to fit long-term Canadian bond prices, as its factor loadings for the slope and curvature factor decay to zero as maturity approaches infinity. This often implies (for reasonable values of $\lambda$ ) that only the level factor in the AFNS model can be used to fit long-term bonds, which may at times be insufficient as noted in Christensen et al. (2011).

To explore whether the performance of the AFNS model on our Canadian sample may be improved further, we briefly consider the generalized AFNS model of Christensen et al. (2009), which includes an additional pair of slope and curvature factors that help to explain long-term bonds. In this AFGNS model, the instantaneous risk-free rate is given by

$$
r_{t}=L_{t}+S_{t}+\tilde{S}_{t}
$$

\footnotetext{
${ }^{21}$ This additional hump is captured by $\beta_{3}(t)$ in equation (12) in Appendix B, which formally presents the Svensson (1995) curve.
} 


\begin{tabular}{c|c|cc|cc|cc}
\hline \hline \multirow{2}{*}{$\begin{array}{c}\text { Maturity } \\
\text { bucket }\end{array}$} & \multirow{2}{*}{$\begin{array}{l}\text { No. } \\
\text { obs. }\end{array}$} & \multicolumn{2}{|c|}{ One-step approach } & \multicolumn{4}{|c}{ Two-step approach } \\
\cline { 3 - 8 } & & Mean & RMSE & Mean & RMSE & Mean & RMSE \\
\hline $0-2$ & 1,472 & -0.41 & 5.51 & 2.91 & 7.84 & 0.70 & 9.54 \\
$2-4$ & 1,098 & 0.47 & 4.92 & 1.55 & 6.92 & 1.26 & 5.20 \\
$4-6$ & 744 & -0.36 & 4.17 & 0.14 & 4.67 & -2.06 & 5.08 \\
$6-8$ & 404 & -1.79 & 5.82 & -1.04 & 5.55 & -3.62 & 7.07 \\
$8-10$ & 477 & -3.65 & 6.75 & -3.04 & 6.89 & -4.76 & 7.77 \\
$10-12$ & 289 & -2.35 & 6.80 & -1.23 & 8.55 & -1.80 & 8.02 \\
$12-14$ & 155 & 2.54 & 5.62 & 4.93 & 11.28 & 5.12 & 10.55 \\
$14-16$ & 168 & -0.34 & 4.17 & -0.96 & 8.66 & 0.89 & 7.90 \\
$16-18$ & 179 & -0.30 & 4.79 & -1.68 & 9.93 & 1.13 & 8.80 \\
$18-20$ & 192 & 0.94 & 3.93 & 1.89 & 8.33 & 4.66 & 8.26 \\
$20-22$ & 186 & 3.31 & 5.38 & 5.44 & 9.58 & 7.43 & 10.07 \\
$22-24$ & 142 & 1.49 & 5.38 & 2.90 & 6.82 & 4.74 & 6.89 \\
$24-26$ & 124 & 1.45 & 5.40 & 4.16 & 8.20 & 4.78 & 7.37 \\
$26-28$ & 113 & -2.98 & 6.88 & 1.14 & 5.66 & 0.43 & 4.45 \\
$28<$ & 288 & -2.59 & 9.24 & 1.16 & 5.95 & -1.71 & 4.59 \\
\hline All yields & 6,031 & -0.52 & 5.62 & 1.15 & 7.34 & 0.15 & 7.57 \\
\hline \hline
\end{tabular}

Table 6: Summary Statistics of Bond Fitted Errors in the B-AFNS Model

This table reports the mean pricing errors (Mean) and the root mean-squared pricing errors (RMSE) of the Canadian bond prices for the B-AFNS model with independent factors. The pricing errors are reported in basis points and computed as the difference between the implied yield on the coupon bond and the model-implied yield on this bond. The data are monthly and cover the period from January 31, 2000, to April 29, 2016.

where $\tilde{S}_{t}$ is an additional (long-term) slope factor. The state dynamics under the risk-neutral $Q$ measure is given by

$$
\left(\begin{array}{c}
d L_{t} \\
d S_{t} \\
d \tilde{S}_{t} \\
d C_{t} \\
d \tilde{C}_{t}
\end{array}\right)=\left(\begin{array}{ccccc}
0 & 0 & 0 & 0 & 0 \\
0 & \lambda & 0 & -\lambda & 0 \\
0 & 0 & \tilde{\lambda} & 0 & -\tilde{\lambda} \\
0 & 0 & 0 & \lambda & 0 \\
0 & 0 & 0 & 0 & \tilde{\lambda}
\end{array}\right)\left[\left(\begin{array}{c}
\theta_{1}^{Q} \\
\theta_{2}^{Q} \\
\theta_{3}^{Q} \\
\theta_{4}^{Q} \\
\theta_{5}^{Q}
\end{array}\right)-\left(\begin{array}{c}
L_{t} \\
S_{t} \\
\tilde{S}_{t} \\
C_{t} \\
\tilde{C}_{t}
\end{array}\right)\right] d t+\Sigma d \tilde{W}_{t}
$$

where $\lambda>\tilde{\lambda}>0$ and $\tilde{C}_{t}$ is an additional (long-term) curvature factor. Zero-coupon yields are then given by

$$
\begin{aligned}
y(t, T)= & L_{t}+\frac{1-e^{-\lambda(T-t)}}{\lambda(T-t)} S_{t}+\left[\frac{1-e^{-\lambda(T-t)}}{\lambda(T-t)}-e^{-\lambda(T-t)}\right] C_{t} \\
& +\frac{1-e^{-\tilde{\lambda}(T-t)}}{\tilde{\lambda}(T-t)} \tilde{S}_{t}+\left[\frac{1-e^{-\tilde{\lambda}(T-t)}}{\tilde{\lambda}(T-t)}-e^{-\tilde{\lambda}(T-t)}\right] \tilde{C}_{t}-\frac{\tilde{A}(t, T)}{T-t},
\end{aligned}
$$




\begin{tabular}{|c|c|c|c|c|c|c|}
\hline \multirow{3}{*}{ Par. } & \multirow{2}{*}{\multicolumn{2}{|c|}{ One-step approach }} & \multicolumn{4}{|c|}{ Two-step approach } \\
\hline & & & \multicolumn{2}{|c|}{ Bank of Canada yields } & \multicolumn{2}{|c|}{ Svensson (1995) yields } \\
\hline & Est & $\mathrm{SE}$ & Est & $\mathrm{SE}$ & Est & $\mathrm{SE}$ \\
\hline$\kappa_{11}^{P}$ & 0.0453 & 0.0484 & 0.3656 & 0.4340 & 0.1365 & 0.1194 \\
\hline$\kappa_{22}^{P}$ & 0.1835 & 0.1418 & 0.6233 & 0.3229 & 0.5279 & 0.2508 \\
\hline$\kappa_{33}^{P}$ & 0.2015 & 0.1973 & 0.1214 & 0.1790 & 1.1160 & 0.3580 \\
\hline$\kappa_{44}^{P}$ & 0.7371 & 0.3144 & 0.9582 & 0.4657 & 1.1599 & 0.3570 \\
\hline$\kappa_{55}^{P}$ & 0.1970 & 0.1255 & 0.3950 & 0.2917 & 0.1464 & 0.1347 \\
\hline$\sigma_{11}$ & 0.0031 & 0.0006 & 0.0092 & 0.0007 & 0.0050 & 0.0002 \\
\hline$\sigma_{22}$ & 0.0125 & 0.0010 & 0.0125 & 0.0009 & 0.0140 & 0.0009 \\
\hline$\sigma_{33}$ & 0.0106 & 0.0009 & 0.0093 & 0.0009 & 0.0150 & 0.0015 \\
\hline$\sigma_{44}$ & 0.0237 & 0.0019 & 0.0209 & 0.0013 & 0.0359 & 0.0021 \\
\hline$\sigma_{55}$ & 0.0200 & 0.0014 & 0.0215 & 0.0033 & 0.0189 & 0.0013 \\
\hline$\theta_{1}^{P}$ & 0.0500 & 0.0053 & 0.0982 & 0.0110 & 0.0580 & 0.0091 \\
\hline$\theta_{2}^{P}$ & 0.0183 & 0.0172 & -0.0040 & 0.0087 & 0.0069 & 0.0099 \\
\hline$\theta_{3}^{P}$ & -0.0452 & 0.0140 & -0.0691 & 0.0219 & -0.0456 & 0.0045 \\
\hline$\stackrel{\stackrel{P}{P}}{\theta_{4}}$ & 0.0064 & 0.0090 & -0.0041 & 0.0071 & 0.0061 & 0.0093 \\
\hline$\theta_{5}^{P}$ & 0.0486 & 0.0185 & -0.0318 & 0.0229 & 0.0252 & 0.0329 \\
\hline$\lambda$ & 0.6416 & 0.0280 & 1.3699 & 0.0297 & 0.9290 & 0.0102 \\
\hline$\tilde{\lambda}$ & 0.1166 & 0.0084 & 0.0786 & 0.0039 & 0.1185 & 0.0026 \\
\hline
\end{tabular}

\section{Table 7: Parameter Estimates in the AFGNS Model}

This table reports the estimated parameters (Est) in the AFGNS model with independent factors and their standard errors (SE) using either the one-step or the two-step approach. The SE in the one-step approach are computed by pre- and post-multiplying the variance of the score by the inverse of the Hessian matrix, as outlined in Harvey (1989). The SE in the two-step approach are computed from the inverse of the variance of the score. The data are monthly and cover the period from January 31 , 2000, to April 29, 2016.

where the yield-adjustment term $\tilde{A}(t, T)$ is derived in Christensen et al. (2009). The $P$ dynamics for this five-factor model is obtained in a standard fashion by adopting an essential affine specification for the market price of risk, as in Section 3.1.

The estimation results for the AFGNS model are reported in Table 7, where we for consistency with the AFNS model only study the case with independent factor dynamics under the $P$-measure. The decay parameter $\lambda$ is estimated to be somewhat larger than in the AFNS model, because $S_{t}$ and $C_{t}$ no longer have to fit long-term bonds. The very low estimate of the second decay parameter $\tilde{\lambda}$ implies that the additional factors $\tilde{S}_{t}$ and $\tilde{C}_{t}$ greatly assist the level factor in matching the long end of the Canadian yield curve.

Table 8 reports the pricing errors of the AFGNS model for the underlying coupon bonds, and we clearly see that all three versions of this five-factor model provide a closer fit to nearly all bonds when compared with the AFNS model. This is highligthed in Table 8 by $\Delta$ RMSE, which shows the difference in RMSE between the AFGNS model and the AFNS model within 


\begin{tabular}{|c|c|c|c|c|c|c|c|c|c|c|}
\hline \multirow{3}{*}{$\begin{array}{c}\text { Maturity } \\
\text { bucket }\end{array}$} & \multirow{3}{*}{$\begin{array}{l}\text { No. } \\
\text { obs. }\end{array}$} & \multirow{2}{*}{\multicolumn{3}{|c|}{ One-step approach }} & \multicolumn{6}{|c|}{ Two-step approach } \\
\hline & & & & & \multicolumn{3}{|c|}{ Bank of Canada yields } & \multicolumn{3}{|c|}{ Svensson (1995) yields } \\
\hline & & Mean & RMSE & $\Delta \mathrm{RMSE}$ & Mean & RMSE & $\Delta \mathrm{RMSE}$ & Mean & RMSE & $\Delta \mathrm{RMSE}$ \\
\hline $0-2$ & 1,472 & -0.20 & 4.86 & -0.88 & 2.63 & 5.56 & -2.31 & -0.73 & 9.01 & -0.80 \\
\hline $2-4$ & 1,098 & 0.36 & 3.82 & -0.92 & -0.93 & 4.49 & -2.71 & 0.33 & 4.28 & -1.33 \\
\hline $4-6$ & 744 & 0.77 & 3.77 & -0.08 & -0.03 & 3.29 & -1.08 & 0.06 & 3.91 & -0.70 \\
\hline $6-8$ & 404 & -0.78 & 3.88 & -1.59 & 1.54 & 3.96 & -1.06 & -1.47 & 4.32 & -2.29 \\
\hline $8-10$ & 477 & -2.39 & 5.33 & -0.74 & 0.91 & 5.00 & -1.61 & -2.28 & 5.31 & -2.50 \\
\hline $10-12$ & 289 & -2.01 & 6.45 & 0.25 & 2.14 & 7.98 & -0.58 & -1.68 & 6.00 & -2.35 \\
\hline $12-14$ & 155 & 1.87 & 4.01 & -2.71 & 7.45 & 10.56 & -1.42 & 2.59 & 4.86 & -6.32 \\
\hline $14-16$ & 168 & 0.03 & 2.45 & -1.87 & 1.71 & 6.36 & -2.69 & 0.61 & 3.04 & -5.24 \\
\hline $16-18$ & 179 & -0.21 & 3.10 & -1.56 & -1.62 & 7.70 & -2.19 & -0.06 & 4.13 & -4.71 \\
\hline $18-20$ & 192 & 0.71 & 3.77 & -0.56 & -0.53 & 5.92 & -2.97 & 0.44 & 4.41 & -4.23 \\
\hline $20-22$ & 186 & 2.39 & 5.10 & 0.13 & 1.17 & 4.74 & -5.32 & 2.47 & 4.69 & -5.63 \\
\hline $22-24$ & 142 & 1.82 & 5.31 & 0.84 & -0.92 & 4.51 & -2.76 & 0.99 & 3.67 & -3.42 \\
\hline $24-26$ & 124 & 1.26 & 4.48 & -0.53 & 0.16 & 5.54 & -2.83 & 1.44 & 3.66 & -3.77 \\
\hline $26-28$ & 113 & -1.21 & 4.51 & -3.85 & -1.51 & 4.71 & -1.19 & -1.56 & 3.51 & -0.98 \\
\hline $28<$ & 288 & -1.88 & 5.32 & -6.59 & 11.59 & 32.98 & 14.85 & 0.33 & 7.82 & -0.87 \\
\hline All bonds & 6,031 & -0.13 & 4.51 & -1.28 & 1.46 & 8.93 & 0.62 & -0.26 & 6.05 & -1.85 \\
\hline
\end{tabular}

Table 8: Summary Statistics of Bond Fitted Errors in the AFGNS Model

This table reports the mean pricing errors (Mean) and the root mean-squared pricing errors (RMSE) of Canadian coupon bond prices for the AFGNS model with independent factors. The table also reports the difference in RMSE ( $\triangle$ RMSE) between the AFGNS model and the AFNS model within the one-step approach and each of the two implementations of the two-step approach. All pricing errors are reported in basis points and computed as the difference between the implied yield on the coupon bond and the model-implied yield on this bond. The data are monthly and cover the period from January 31, 2000, to April 29, 2016.

the one-step approach and each of the two implementations of the two-step approach. For the one-step approach, we see large improvements in the RMSEs for long-term bonds, but also in the zero to two-year and two- to four-year maturity buckets, which both contain a large numbers of bonds. As a result, the overall RMSE within the one-step approach drops from 5.79 basis points in the AFNS model to just 4.51 basis points in this extended model, which corresponds to a $22 \%$ reduction in the size of the in-sample fitted errors. This also means that the AFGNS model clearly provides a better overall fit to bond prices than the Svensson (1995) discount function with an overall RMSE of 5.78 basis points. Given this satisfying performance of the AFGNS model, its zero-coupon yields may thus be used as another and slightly more accurate representation of the Canadian yield curve than the zero-coupon yields from the Svensson (1995) discount function.

Accordingly, allowing for an additional pair of slope and curvature factors improves the ability of the AFNS model to fit bond prices, in particular those of long-term bonds. 


\section{Simulation Study}

The preceding analysis has shown that the one-step and the two-step approach give somewhat different estimates of DTSMs. The obvious next question is which of the two approaches gives the most accurate estimates? The present section addresses this question by conducting a Monte Carlo study to analyze the finite sample properties of estimating the AFNS model by the one-step and two-step approaches. We first describe the setup for the Monte Carlo study in Section 5.1 before analyzing the precision of the estimated synthetic zero-coupon yields from the Svensson (1995) yield curve in Section 5.2. The results for the estimated model parameters are reported in Section 5.3, while the accuracy of the filtered states and the standard yield curve decomposition are explored in Section 5.4 and 5.5, respectively. Section 5.6 is devoted to the implementation of the two-step approach, where we explore how the number of synthetic yields and the adopted curve-fitting technique for these yields affect the estimated parameters in our DTSM. Finally, Section 5.7 summarizes the main findings from this Monte Carlo study.

\subsection{Setup for the Monte Carlo Study}

A novel feature of this Monte Carlo study is to work at the level of individual coupon bond prices, and hence account for estimation uncertainty in the construction of synthetic zerocoupon yields within the two-step approach. To get a representative data generating process for the Canadian bond market, we use the estimates of the AFNS model in the one-step approach from Table 2. Based on these parameters, we first simulate $N=100$ samples for the three states at a monthly frequency for 196 months, which corresponds to the number of monthly observations in our Canadian sample. ${ }^{22}$ These simulated sample paths will be common across all exercises in this Monte Carlo study to facilitate the interpretation. The inputs for each of the two estimation approaches are then constructed as follows.

For the one-step approach, we use the simulated states to compute $N$ panels of couponbond prices that match those observed in the Canadian sample in terms of available bonds and their characteristics. These bond prices are computed using the bond price formula in equation (5) in combination with the zero-coupon yields in equation (3). We then add measurement errors $\varepsilon_{t}^{i} \sim \mathcal{N} \mathcal{I D}\left(0, \sigma_{\varepsilon}^{2}\right)$ to the individual simulated bond prices and scale these measurement errors by the duration of the simulated bond for consistency with equation (6). ${ }^{23}$

For the two-step approach, we take these simulated panels of coupon bond prices as

\footnotetext{
${ }^{22}$ We simulate from (4) using a standard Euler-discretization, i.e., $X_{t}^{i}=X_{t-1}^{i}+\kappa_{i i}^{P}\left(\theta_{i}^{P}-X_{t-1}^{i}\right) \Delta t+\sigma_{i i} \sqrt{\Delta t} z_{t}^{i}$, where $z_{t}^{i} \sim N(0,1)$ and $\Delta t=0.0001$. The starting values $X_{0}^{i}$ are drawn from the unconditional distribution of $X_{t}$.

${ }^{23}$ Note that we use the same set of simulated samples of $\varepsilon_{t}^{i}$ throughout this Monte Carlo study to make the results as comparable as possible.
} 
input to extract synthetic zero-coupon yields based on the Svensson (1995) yield curve. For consistency with the empirical estimation results presented in the previous sections, we extract synthetic zero-coupon yields with ten constant maturities, $0.25,0.5,1,2,3,5,7,10$, 20, and 30 years, which we use for our implementation of the two-step approach in the Monte Carlo study. Given that the underlying bond prices are already contaminated with measurement errors, we do not add additional noise to these synthetic yields.

To study the role of the data quality, we consider two cases where $\sigma_{\varepsilon}$ is either 1 or 10 basis points. The first case with $\sigma_{\varepsilon}=1$ basis point represents an ideal setting with hardly any noise in bond prices and helps to isolate the effects of the curve fitting procedure in the two-step approach. The second case with $\sigma_{\varepsilon}=10$ basis points is included to describe a more realistic setting, as we find that $\sigma_{\varepsilon}=7$ basis points in our Canadian sample when using the one-step approach.

\subsection{Accuracy of Synthetic Yields}

We first consider the accuracy of the synthetic zero-coupon yields from the Svensson (1995) yield curve based on the simulated prices for coupon bonds. That is, we compare the estimated synthetic yields to the true zero-coupon yields from the AFNS model without measurement errors, see Appendix B for details.

With small measurement errors of $\sigma_{\varepsilon}=1$ basis point, Table 9 shows that the mean errors are generally very close to zero within the one- to twenty-year maturity range but somewhat larger at the three- and six-month maturities (-4 and -2 basis points, respectively) and at the thirty-year maturity (-1.3 basis points). This means that the Svensson (1995) yield curve slightly overpredicts the level of the zero-coupon yields at the short and long end of the curve. The low mean absolute errors (MAE) of roughly 1 basis point show that yields within the one- to twenty-year maturity spectrum are estimated very accurately, whereas yields at the short and long end of the curve are estimated less precisely. For instance, we have a MAE of 6.8 basis points for the three-month yield and a MAE of 3.09 basis points for the thirty-year yield, as the short and long end of the curve is not always densely populated in the underlying panels of bond prices.

With larger measurement errors of $\sigma_{\varepsilon}=10$ basis points, maturities between one and twenty years remain well approximated with mean errors close to zero. The precision of these yields in terms of the MAEs only decreases by a factor of three, which is substantially lower than the ten-fold increase in $\sigma_{\varepsilon}$. Thus, when $\sigma_{\varepsilon}=10$ basis points, the construction of synthetic zero-coupon yields is in most cases able to smooth out a large fraction of the noise from $\varepsilon_{t}^{i}$ in the underlying bond prices, which generally leaves the measurement equation in the two-step approach with smaller measurement errors than in the one-step approach. Hence, for $\sigma_{\varepsilon}=10$ basis points, the considered setup is likely to favor the two-step approach, 


\begin{tabular}{c|c|c|c|c}
\hline \hline Maturity & \multicolumn{2}{|c|}{$\sigma_{\varepsilon}=1$} & basis point & \multicolumn{2}{c}{$\sigma_{\varepsilon}=10$ basis points } \\
\cline { 2 - 5 } in months & Mean & MAE & Mean & MAE \\
\hline 3 & -4.09 & 6.79 & -3.00 & 12.48 \\
6 & -2.37 & 3.89 & -1.74 & 7.84 \\
12 & -0.32 & 1.05 & -0.24 & 3.58 \\
24 & 0.78 & 1.42 & 0.52 & 3.52 \\
36 & 0.43 & 0.99 & 0.24 & 3.12 \\
60 & -0.48 & 1.05 & -0.34 & 3.13 \\
84 & -0.67 & 1.26 & -0.37 & 3.30 \\
120 & -0.34 & 0.94 & -0.14 & 2.88 \\
240 & 0.64 & 1.46 & 0.27 & 3.76 \\
360 & -1.32 & 3.09 & -0.90 & 9.34 \\
\hline \hline
\end{tabular}

Table 9: Accuracy of Estimated Svensson (1995) Yields

The table reports the mean of the sampling distribution of the mean errors (Mean) and mean absolute errors (MAE) for each zero-coupon yield constructed using the Svensson (1995) yield curve relative to the true zero-coupon yield implied by the AFNS model with simulated samples of length $T=196$ and $N=100$ repetitions. The mean is obtained by first computing the mean errors in each of the simulated samples across the $T=196$ observations, and we then report the average of these means across the $N=100$ simulated samples. Similarly, the MAE are obtained by first computing the mean absolute errors in each of the simulated samples across the $T=196$ observations, and we then report the average of these absolute means across the $N=100$ simulated samples. The true states are generated from the AFNS model as described in Section 5.1. All numbers are reported in basis points.

as the measurement equation here has less noise than in the one-step approach.

Accordingly, the synthetic zero-coupon yields from the Svensson (1995) yield curve appear to be quite accurate in the present setting, although with slightly larger errors at the short and long end of the yield curve.

\subsection{Estimated Parameters}

Table 10 summarizes the outcome of the Monte Carlo study for the model parameters by reporting the mean and the standard deviation for each of the estimated coefficients in the AFNS model. We first note that both the one-step and the two-step approach generate the familiar positive bias in the mean-reversion parameters $\left\{\kappa_{11}^{P}, \kappa_{22}^{P}, \kappa_{33}^{P}\right\}$, as discussed in Bauer et al. (2012). For the persistence of the slope factor $\kappa_{22}^{P}$ and the curvature factor $\kappa_{33}^{P}$, we find that these biases are somewhat larger in the two-step approach compared with the one-step approach. For instance, with $\sigma_{\varepsilon}=10$ basis points, the bias in the slope factor is $0.51-0.22=0.29$ in the one-step approach but $0.66-0.22=0.44$ in the two-step approach. The corresponding figures for the curvature factor are $0.93-0.73=0.20$ for the one-step approach and $1.30-0.73=0.57$ for the two-step approach. However, we see the opposite pattern in $\kappa_{11}^{P}$, as the bias here is slightly smaller for the conventional two-step approach 


\begin{tabular}{c|c|c|c|c|c||c|c|c|c}
\hline \hline \multirow{2}{*}{ Par. } & \multirow{2}{*}{ True } & \multicolumn{4}{|c||}{ One-step approach } & \multicolumn{4}{c}{ Two-step approach } \\
\cline { 3 - 10 } & value & $\sigma_{\varepsilon}=1$ basis point & $\sigma_{\varepsilon}=10$ basis points & \multicolumn{2}{c}{$\sigma_{\varepsilon}=1$ basis point } & $\sigma_{\varepsilon}=10$ basis points \\
\cline { 3 - 10 } & & Mean & Std. dev. & Mean & Std. dev. & Mean & Std. dev. & Mean & Std. dev. \\
\hline$\kappa_{11}^{P}$ & $\mathbf{0 . 1 0 6 0}$ & 0.3794 & 0.2660 & 0.4011 & 0.2659 & 0.3457 & 0.2114 & 0.3549 & 0.2186 \\
$\kappa_{22}^{P}$ & $\mathbf{0 . 2 1 5 7}$ & 0.5127 & 0.3022 & 0.5096 & 0.3002 & 0.5296 & 0.3069 & 0.6611 & 0.3883 \\
$\kappa_{33}^{P}$ & $\mathbf{0 . 7 2 5 5}$ & 0.9056 & 0.3973 & 0.9298 & 0.3706 & 1.1519 & 0.6491 & 1.3011 & 0.5329 \\
\hline$\sigma_{11}$ & $\mathbf{0 . 0 0 5 2}$ & 0.0052 & 0.0000 & 0.0052 & 0.0001 & 0.0047 & 0.0003 & 0.0048 & 0.0003 \\
$\sigma_{22}$ & $\mathbf{0 . 0 1 0 3}$ & 0.0103 & 0.0005 & 0.0103 & 0.0006 & 0.0105 & 0.0006 & 0.0117 & 0.0009 \\
$\sigma_{33}$ & $\mathbf{0 . 0 2 0 7}$ & 0.0206 & 0.0007 & 0.0208 & 0.0014 & 0.0242 & 0.0037 & 0.0265 & 0.0020 \\
\hline$\theta_{1}^{P}$ & $\mathbf{0 . 0 5 2 9}$ & 0.0514 & 0.0088 & 0.0510 & 0.0086 & 0.0506 & 0.0086 & 0.0513 & 0.0087 \\
$\theta_{2}^{P}$ & $\mathbf{- 0 . 0 2 7 5}$ & -0.0254 & 0.0108 & -0.0255 & 0.0108 & -0.0239 & 0.0112 & -0.0247 & 0.0110 \\
$\theta_{3}^{P}$ & $\mathbf{- 0 . 0 2 3 0}$ & -0.0242 & 0.0098 & -0.0236 & 0.0064 & -0.0257 & 0.0062 & -0.0261 & 0.0062 \\
\hline$\lambda$ & $\mathbf{0 . 3 7 4 7}$ & 0.3747 & 0.0008 & 0.3743 & 0.0056 & 0.4237 & 0.0314 & 0.4170 & 0.0264 \\
\hline \hline
\end{tabular}

Table 10: Accuracy of the Parameter Estimates in the AFNS Model

The table reports the mean (Mean) and the standard deviation (Std. dev.) of the sampling distribution for each of the estimated parameters in the AFNS model when using QML in the one-step approach and ML in the two-step approach, where synthetic yields are generated with the Svensson (1995) yield curve, both with simulated samples of length $T=196$ and $N=100$ repetitions.

compared with the one-step approach.

The estimates of the volatility parameters in $\Sigma$ are basically unbiased in the one-step approach and estimated with great precision - both with small and large measurement errors. The corresponding estimates in the two-step approach display small biases with $\sigma_{\varepsilon}=1$ basis points, which generally increase with larger measurement errors. For instance, $\sigma_{22}$ in the two-step approach displays a positive bias of $0.0242-0.0207=0.0035$ when $\sigma_{\varepsilon}=1$ basis point, but this bias increases to $0.0265-0.0207=0.0058$ with $\sigma_{\varepsilon}=10$ basis points.

All elements in $\theta^{P}$ are generally close to their true values, although a careful inspection of Table 10 reveals that the biases in $\theta^{P}$ typically are smaller in the one-step approach compared with the two-step approach.

The estimates of the decay parameter $\lambda$ for the slope and curvature factor are centered exactly around its true value in the one-step approach and estimated with great precisionboth with small and large measurement errors. For the two-step approach, we see small positive biases in the estimates of $\lambda$, which is estimated less precisely compared to the onestep approach. For instance, when $\sigma_{\varepsilon}=10$ basis points, the standard deviation in the estimates of $\lambda$ are 0.0264 in the two-step approach, but only 0.0056 in the one-step approach. These differences in the estimates of $\lambda$ are of particular interest given the work of Björk and Christensen (1999), which shows that the static Nelson-Siegel and Svensson yield curves are inconsistent with no-arbitrage restrictions because the corresponding $\lambda$ parameter(s) in these static models may change across time. The biased estimate of $\lambda$ in the two-step approach implies that the curvature factor carries a greater weight on shorter-term yields and is less sensitive to longer-term yields relative to the true model. Since short-term yields are more 


\begin{tabular}{c|c|c|c|c||c|c|c|c}
\hline \hline \multirow{2}{*}{$\begin{array}{c}\text { State } \\
\text { variable }\end{array}$} & \multicolumn{4}{|c||}{ One-step approach } & \multicolumn{4}{c}{ Two-step approach } \\
\cline { 2 - 8 } & $\sigma_{\varepsilon}=1$ basis point & $\sigma_{\varepsilon}=10$ basis points & \multicolumn{4}{c}{$\sigma_{\varepsilon}=1$ basis point } & $\sigma_{\varepsilon}=10$ basis points \\
\cline { 2 - 8 } & Mean & MAE & Mean & MAE & Mean & MAE & Mean & MAE \\
\hline$L_{t}$ & -0.41 & 1.16 & 0.21 & 5.51 & -8.93 & 10.80 & -3.05 & 9.66 \\
$S_{t}$ & 0.42 & 1.16 & -0.14 & 5.97 & 14.28 & 15.67 & 7.45 & 13.46 \\
$C_{t}$ & 0.16 & 2.51 & -0.58 & 19.07 & -21.70 & 32.33 & -25.06 & 42.62 \\
\hline \hline
\end{tabular}

Table 11: Accuracy of Estimated States in the AFNS Model

The table reports the mean of the sampling distribution of the mean errors (Mean) and mean absolute errors (MAE) of each estimated state variable in the AFNS model when using QML in the one-step approach and ML in the two-step approach, where synthetic yields are generated with the Svensson (1995) yield curve, both with simulated samples of length $T=196$ and $N=100$ repetitions. The mean is obtained by first computing the mean errors in each of the simulated samples across the $T=196$ observations, and we then report the average of these means across the $N=100$ simulated samples. Similarly, the MAE are obtained by first computing the mean absolute errors in each of the simulated samples across the $T=196$ observations, and we then report the average of these absolute means across the $N=100$ simulated samples. The true states are generated from the AFNS model as described in Section 5.1. All numbers are reported in basis points.

volatile than long-term yields, this explains the positive bias in the estimates of $\sigma_{33}$ within the two-step approach. Furthermore, since short-term yields also tend to be less persistent than long-term yields, this also explains the more severe upward bias in the estimates of $\kappa_{33}^{P}$ in the two-step approach compared with the one-step approach.

\subsection{Estimated States}

For each simulated sample and its related set of estimated parameters, we next study the accuracy of the filtered states. Table 11 shows that the filtered states in the one-step approach are basically unbiased, as the mean errors with $\sigma_{\varepsilon}=10$ basis points are $0.21,-0.14$, and -0.58 basis points for the level, slope, and curvature factor, respectively. In contrast, the conventional two-step approach generates notable biases in the estimated states, which are $-3,7$, and -25 basis points for the level, slope, and curvature factor, respectively, with $\sigma_{\varepsilon}=10$ basis points. We also note that these biases in the two-step approach are largely unrelated to the degree of noise in the bond prices, implying that these biases must originate from the use of the estimated synthetic zero-coupon yields.

To measure the efficiency of the filtered states, we compute the mean absolute errors in each simulated sample of $T=196$ observations, which we then report in Table 11 by averaging across the $N=100$ simulations. The states in the one-step approach are estimated very accurately with mean absolute errors of 1 to 2 basis points in the ideal case with $\sigma_{\varepsilon}=1$ basis point. For the more realistic setting where $\sigma_{\varepsilon}=10$ basis points, we find somewhat larger mean absolute errors of 6, 6, and 19 basis points for the level, slope, and curvature factor, respectively. The filtered states in the two-step approach are estimated much less accurately 
due to its lower number of cross-sectional observations to represent the yield curve compared with the one-step approach. For instance, the mean absolute errors are 10, 13, and 43 basis points for the level, slope, and curvature factor, respectively, in the two-step approach with $\sigma_{\varepsilon}=10$ basis points. It is also interesting to note that the efficiency of the state estimates in the two-step approach are much less affected by increasing the noise in bond prices than seen in the one-step approach. For instance, the mean absolute errors of the level and slope factor are basically unaffected by the value of $\sigma_{\varepsilon}$. This feature of the two-step approach reflects the fact that the synthetic zero-coupon yields are able to smooth out much of the noise in the bond prices, and in this way mitigate the effects of measurement errors.

\subsection{Accuracy of Yield Decomposition}

DTSMs are often applied to decompose the yield curve into expected future short rates and term premiums. We next explore whether the use of the one-step approach improves the precision of this decomposition compared to the conventional two-step approach. Hence, let the $\tau$ year term premium be defined as $T P_{t}(\tau)=y\left(\tau, X_{t}\right)-\frac{1}{\tau} \int_{t}^{t+\tau} E_{t}^{P}\left[r_{s}\right] d s$, where $\frac{1}{\tau} \int_{t}^{t+\tau} E_{t}^{P}\left[r_{s}\right] d s$ denotes expected future short rates. ${ }^{24}$

For each simulated sample and its related set of estimated parameters and states, we next decompose the yield curve into expected future short rates and term premiums in Table 12. We first note that the mean errors in expected future short rates (EXP) are somewhat closer to zero in the one-step approach than in to the two-step approach. For instance, at the ten-year maturity with $\sigma_{\varepsilon}=10$ basis points, the mean errors in expected future short rates are -3.64 basis points in the one-step approach but -11.02 basis points in the two-step approach. This finding seems consistent with the smaller biases in $K^{P}$ and the filtered states for the one-step approach relative to the two-step approach reported in Section 5.3 and 5.4. Given the small errors in estimating yields in both approaches, we therefore find that the onestep approach implies slightly lower mean errors for term premiums (TP) than the two-step approach. However, the mean absolute errors (MAE) in Table 12 for expected future short rates and term premiums are very large and almost identical for both estimation approaches, meaning that the large estimation uncertainty clearly dominates the small improvement in mean errors for term premiums within the one-step approach.

Thus, standard yield curve decomposition do not benefit from the proposed one-step approach. This is because the reported biases and large estimation uncertainty in term premiums originate from expected future short rates, and hence the estimated mean-reversion parameters in $K^{P}$, which are relatively insensitive to the number of cross-sectional observations used to represent the yield curve.

\footnotetext{
${ }^{24} \mathrm{~A}$ closed-form expression for $\frac{1}{\tau} \int_{t}^{t+\tau} E_{t}^{P}\left[r_{s}\right] d s$ is derived in Appendix F.
} 


\begin{tabular}{l|c|c|c|c||c|c|c|c}
\hline \hline \multirow{2}{*}{ Component } & \multicolumn{4}{|c||}{ One-step approach } & \multicolumn{4}{c}{ Two-step approach } \\
\cline { 2 - 9 } & $\sigma_{\varepsilon}=1$ basis point & $\sigma_{\varepsilon}=10$ basis points & $\sigma_{\varepsilon}=1$ basis point & $\sigma_{\varepsilon}=10$ basis points \\
\cline { 2 - 9 } & Mean & MAE & Mean & MAE & Mean & MAE & Mean & MAE \\
\hline Two-year yield & 0.09 & 0.24 & 0.08 & 2.04 & 0.66 & 1.23 & 0.77 & 2.80 \\
Five-year yield & 0.18 & 0.33 & 0.19 & 2.23 & -0.21 & 0.88 & -0.23 & 2.67 \\
Ten-year yield & 0.25 & 0.33 & 0.24 & 1.72 & -0.57 & 1.40 & -0.67 & 2.67 \\
\hline Two-year EXP & -1.96 & 24.09 & -1.67 & 24.27 & -7.71 & 24.60 & -7.08 & 27.81 \\
Five-year EXP & -3.76 & 42.51 & -2.95 & 42.15 & -9.85 & 41.25 & -9.36 & 43.84 \\
Ten-year EXP & -5.20 & 59.37 & -3.64 & 58.49 & -11.55 & 56.81 & -11.02 & 58.67 \\
\hline Two-year TP & 2.05 & 24.09 & 1.75 & 24.23 & 8.36 & 24.64 & 7.85 & 27.64 \\
Five-year TP & 3.95 & 42.52 & 3.13 & 42.33 & 9.64 & 41.36 & 9.13 & 44.16 \\
Ten-year TP & 5.44 & 59.38 & 3.88 & 58.53 & 10.98 & 56.85 & 10.34 & 58.72 \\
\hline \hline
\end{tabular}

Table 12: Accuracy of the Yield Decomposition in the AFNS Model

The table reports the mean of the sampling distribution of the mean errors (Mean) and mean absolute errors (MAE) for each component of the yield curve decomposition into expected future short rates (EXP) and term premium (TP) at various maturities. The mean for a given maturity is obtained by first computing the mean errors in each of the simulated samples across the $T=196$ observations, and we then report the average of these means across the $N=100$ simulated samples. Similarly, the MAE for a given maturity is obtained by first computing the mean absolute errors in each of the simulated samples across the $T=196$ observations, and we then report the average of these absolute means across the $N=100$ simulated samples. All errors are shown in basis points and defined as the true value minus the model-implied value. The parameter and state estimates in the AFNS model are obtained by QML in the one-step approach and by ML in the two-step approach, where zero-coupon synthetic yields are generated with the Svensson (1995) yield curve. The true yields and expected future short rates are generated from the AFNS model as described in Section 5.1.

\subsection{The Implementation of the Two-Step Approach}

Given the widespread use of the conventional two-step approach, it seems useful to explore whether its performance can be improved compared to Sections 5.3 and 5.4. The essential decisions for the econometrician in the two-step approach are how many synthetic zero-coupon yields to include when estimating the DTSM, and how these synthetic yields are extracted from the panel of coupon bonds. We next analyze how decisions along each of these dimensions affect the estimated parameters in the AFNS model.

\subsubsection{The Number of Synthetic Yields}

Our implementation of the two-step approach has so far used ten synthetic zero-coupon yields to represent the thirty-year Canadian yield curve. But the choice of which synthetic yields to include is likely to affect the performance of the two-step approach. In particular, it is likely that there may exist a trade-off within the two-step approach between bias and efficiency for the estimated parameters in the DTSM. The reason is as follows. To increase efficiency, it is obviously desirable to use a large number of synthetic yields, but this is likely to come 


\begin{tabular}{c|c|c|c|c|c||c|c|c|c}
\hline \hline \multirow{2}{*}{ Par. } & \multirow{2}{*}{$\begin{array}{c}\text { True } \\
\text { value }\end{array}$} & \multicolumn{4}{|c||}{ Extended sample } & \multicolumn{4}{c}{ Reduced sample } \\
\cline { 3 - 10 } & & $\sigma_{\varepsilon}=1$ basis point & $\sigma_{\varepsilon}=10$ basis points & $\sigma_{\varepsilon}=1$ basis point & $\sigma_{\varepsilon}=10$ basis points \\
\cline { 3 - 10 } & & Mean & Std. dev. & Mean & Std. dev. & Mean & Std. dev. & Mean & Std. dev. \\
\hline$\kappa_{11}^{P}$ & $\mathbf{0 . 1 0 6 0}$ & 0.3609 & 0.2245 & 0.3914 & 0.2397 & 0.3802 & 0.2145 & 0.5419 & 0.3017 \\
$\kappa_{22}^{P}$ & $\mathbf{0 . 2 1 5 7}$ & 0.5072 & 0.2961 & 0.5964 & 0.3518 & 0.5209 & 0.2959 & 0.6120 & 0.3488 \\
$\kappa_{33}^{P}$ & $\mathbf{0 . 7 2 5 5}$ & 3.2985 & 2.3665 & 2.5187 & 1.6718 & 0.9266 & 0.4196 & 1.2340 & 0.5102 \\
\hline$\sigma_{11}$ & $\mathbf{0 . 0 0 5 2}$ & 0.0048 & 0.0004 & 0.0051 & 0.0003 & 0.0050 & 0.0004 & 0.0061 & 0.0004 \\
$\sigma_{22}$ & $\mathbf{0 . 0 1 0 3}$ & 0.0103 & 0.0005 & 0.0111 & 0.0007 & 0.0105 & 0.0005 & 0.0114 & 0.0007 \\
$\sigma_{33}$ & $\mathbf{0 . 0 2 0 7}$ & 0.0399 & 0.0135 & 0.0351 & 0.0084 & 0.0195 & 0.0016 & 0.0235 & 0.0017 \\
\hline$\theta_{1}^{P}$ & $\mathbf{0 . 0 5 2 9}$ & 0.0556 & 0.0065 & 0.0550 & 0.0082 & 0.0496 & 0.0084 & 0.0508 & 0.0086 \\
$\theta_{2}^{P}$ & $\mathbf{- 0 . 0 2 7 5}$ & -0.0290 & 0.0118 & -0.0287 & 0.0115 & -0.0230 & 0.0107 & -0.0243 & 0.0108 \\
$\theta_{3}^{P}$ & $\mathbf{- 0 . 0 2 3 0}$ & -0.0299 & 0.0097 & -0.0278 & 0.0083 & -0.0251 & 0.0060 & -0.0260 & 0.0059 \\
\hline$\lambda$ & $\mathbf{0 . 3 7 4 7}$ & 0.3773 & 0.0178 & 0.3614 & 0.0210 & 0.4418 & 0.0308 & 0.4263 & 0.0256 \\
\hline \hline
\end{tabular}

Table 13: Accuracy of the Parameter Estimates: The Number of Synthetic Yields The table reports the mean (Mean) and the standard deviation (Std. dev.) of the sampling distribution for each of the estimated parameters in the AFNS model when using ML in the two-step approach, with simulated samples of length $T=196$ and $N=100$ repetitions. The extended samples consists of the 31 synthetic zero-coupon yields with maturities of $0.5,1,2, \ldots, 30$ year from the Svensson (1995) yield curve. The reduced sample consists of six synthetic zero-coupon yields with maturities of 1,2 , $3,5,7$, and 10 years from the Svensson (1995) yield curve.

at the cost of also including some less precisely measured yields, and this could potentially bias the coefficients in the DTSM. On the other hand, when only a few and very accurately measured yields are included, then we minimize the risk of coefficient bias but at the cost of low efficiency. The present section explores whether such a trade-off exist by implementing the two-step approach on two different sets of samples that both represent the same data generating process. The first is an extended sample of 31 synthetic yields with maturities of $0.5,1,2, \ldots, 30$ year. The second is a reduced sample of only six yields with maturities of 1 , $2,3,5,7$, and 10 years, where we omit the imprecisely estimated yields at the short and long end of the thirty-year yield curve.

The estimated coefficients in the AFNS model from this simulation exercise are reported in Table 13. We find somewhat surprisingly that the biases for $\kappa_{11}^{P}, \kappa_{22}^{P}, \sigma_{11}^{P}, \sigma_{22}^{P}$, and $\lambda$ are smaller in the extended sample compared to the standard sample (in Table 10) and the reduced sample. Still, it is hard to detect any efficiency gains from the extended sample for these parameters, except possibly for $\lambda$. Furthermore, we note that the estimates of $\kappa_{33}^{P}$ and $\sigma_{33}^{P}$ have the largest biases in the extended sample, which also provides the most imprecise estimates of $\kappa_{33}^{P}$ and $\sigma_{33}^{P}$. The performance of the reduced sample with six synthetic yields is very similar to what we found for the standard sample with ten synthetic yields, meaning that the reduced sample avoids the large biases we occasionally find in the extended sample (e.g. in $\kappa_{33}^{P}$ and $\sigma_{33}^{P}$ ).

For each simulated sample and its related set of estimated parameters, Table 14 studies 


\begin{tabular}{c|c|c|c|c||c|c|c|c}
\hline \hline \multirow{2}{*}{$\begin{array}{c}\text { State } \\
\text { variable }\end{array}$} & \multicolumn{4}{|c||}{ Extended sample } & \multicolumn{4}{c}{ Reduced sample } \\
\cline { 2 - 8 } & $\sigma_{\varepsilon}=1$ basis point & \multicolumn{2}{|c||}{$\sigma_{\varepsilon}=10$ basis points } & $\sigma_{\varepsilon}=1$ basis point & $\sigma_{\varepsilon}=10$ basis points \\
\cline { 2 - 8 } & Mean & MAE & Mean & MAE & Mean & MAE & Mean & MAE \\
\hline$L_{t}$ & 40.27 & 41.36 & 34.44 & 34.78 & -19.59 & 20.33 & -7.31 & 14.52 \\
$S_{t}$ & -35.83 & 37.46 & -32.06 & 32.77 & 24.68 & 25.21 & 11.98 & 17.13 \\
$C_{t}$ & -65.82 & 67.28 & -43.46 & 54.12 & -14.52 & 31.40 & -23.80 & 43.47 \\
\hline \hline
\end{tabular}

Table 14: Accuracy of Estimated States: The Number of Synthetic Yields

The table reports the mean of the sampling distribution of the mean errors (Mean) and mean absolute errors (MAE) of each estimated state variable in the AFNS model using ML in the two-step approach. The mean is obtained by first computing the mean errors in each of the simulated samples across the $T=196$ observations, and we then report the average of these means across the $N=100$ simulated samples. Similarly, the MAE are obtained by first computing the mean absolute errors in each of the simulated samples across the $T=196$ observations, and we then report the average of these absolute means across the $N=100$ simulated samples. The true states are generated from the AFNS model as described in Section 5.1. The extended samples consists of the 31 synthetic zero-coupon yields with maturities of $0.5,1,2, \ldots, 30$ year from the Svensson (1995) yield curve. The reduced sample consists of six synthetic zero-coupon yields with maturities of 1, 2, 3, 5, 7, and 10 years from the Svensson (1995) yield curve. All numbers are reported in basis points.

the accuracy of the filtered states. For the extended sample, we find large positive biases for the level factor and large negative biases for the slope and curvature factor. For instance, the mean error for the curvature factor is -66 basis points when $\sigma_{\varepsilon}=10$ basis points. The biases in the reduced sample are much smaller than in the extended sample, and this also explains the lower mean absolute errors (MAE) of the filtered states for the reduced sample compared with the extended sample.

We draw two conclusions from this exercise. First, there does not appear to be an obvious trade-off between bias and efficiency in the two-step approach when varying the number of synthetic zero-coupon yields in the estimation of the DTSM. Second, the current practice of using a relatively low number of synthetic yields (i.e. between six and ten) seems well justified, at least when the synthetic yields are extracted based on the parametric discount function in Svensson (1995).

\subsubsection{Synthetic Nelson and Siegel (1987) Yields}

An obvious difference between the true zero-coupon yields from the AFNS model and those from the Svensson (1995) yield curve is that the latter allows for an extra 'hump' at the long end of the yield curve compared to the AFNS model. Within our setting, this additional hump clearly seems redundant and we therefore explore the effects of omitting it when extracting synthetic zero-coupon yields from our simulated panels of bond prices. That is, we briefly consider the case where the synthetic yields are extracted based on the parametric discount function in Nelson and Siegel (1987), and we continue to use the same ten constant yield 


\begin{tabular}{c|c|c|c|c|c||c|c|c|c}
\hline \hline \multirow{2}{*}{ Par. } & \multirow{2}{*}{ True } & \multicolumn{4}{|c||}{ Svensson (1995) yields } & \multicolumn{4}{c}{ Nelson and Siegel (1987) yields } \\
\cline { 3 - 10 } & value & $\sigma_{\varepsilon}=1$ basis point & $\sigma_{\varepsilon}=10$ basis points & \multicolumn{2}{c}{$\sigma_{\varepsilon}=1$ basis point } & $\sigma_{\varepsilon}=10$ basis points \\
\cline { 3 - 10 } & & Mean & Std. dev. & Mean & Std. dev. & Mean & Std. dev. & Mean & Std. dev. \\
\hline$\kappa_{11}^{P}$ & $\mathbf{0 . 1 0 6 0}$ & 0.3457 & 0.2114 & 0.3549 & 0.2186 & 0.1320 & 0.1009 & 0.1611 & 0.1195 \\
$\kappa_{22}^{P}$ & $\mathbf{0 . 2 1 5 7}$ & 0.5296 & 0.3069 & 0.6611 & 0.3883 & 0.5412 & 0.2813 & 0.6661 & 0.3739 \\
$\kappa_{33}^{P}$ & $\mathbf{0 . 7 2 5 5}$ & 1.1519 & 0.6491 & 1.3011 & 0.5329 & 3.4829 & 2.9780 & 2.0118 & 1.1920 \\
\hline$\sigma_{11}$ & $\mathbf{0 . 0 0 5 2}$ & 0.0047 & 0.0003 & 0.0048 & 0.0003 & 0.0029 & 0.0003 & 0.0033 & 0.0003 \\
$\sigma_{22}$ & $\mathbf{0 . 0 1 0 3}$ & 0.0105 & 0.0006 & 0.0117 & 0.0009 & 0.0107 & 0.0005 & 0.0118 & 0.0008 \\
$\sigma_{33}$ & $\mathbf{0 . 0 2 0 7}$ & 0.0242 & 0.0037 & 0.0265 & 0.0020 & 0.0417 & 0.0179 & 0.0332 & 0.0087 \\
\hline$\theta_{1}^{P}$ & $\mathbf{0 . 0 5 2 9}$ & 0.0506 & 0.0086 & 0.0513 & 0.0087 & 0.0516 & 0.0073 & 0.0502 & 0.0079 \\
$\theta_{2}^{P}$ & $\mathbf{- 0 . 0 2 7 5}$ & -0.0239 & 0.0112 & -0.0247 & 0.0110 & -0.0264 & 0.0127 & -0.0239 & 0.0114 \\
$\theta_{3}^{P}$ & $\mathbf{- 0 . 0 2 3 0}$ & -0.0257 & 0.0062 & -0.0261 & 0.0062 & -0.0281 & 0.0110 & -0.0258 & 0.0074 \\
\hline$\lambda$ & $\mathbf{0 . 3 7 4 7}$ & 0.4237 & 0.0314 & 0.4170 & 0.0264 & 0.4441 & 0.0310 & 0.4525 & 0.0268 \\
\hline \hline
\end{tabular}

Table 15: Accuracy of the Parameter Estimates: Different Synthetic Yields

The table reports the mean (Mean) and the standard deviation (Std. dev.) of the sampling distribution for each of the estimated parameters in the AFNS model when using ML in the two-step approach on synthetic yields from the Svensson (1995) and Nelson and Siegel (1987) yield curves. The true yields are generated from the AFNS model as described in Section 5.1, with simulated samples of length $T=196$ and $N=100$ repetitions. For both types of yields we use the same ten constant maturities, $0.25,0.5,1,2,3,5,7,10,20$, and 30 years.

maturities as in Section 5.3.

Table 15 reports the results for the estimated coefficients in the AFNS model from this simulation exercise, which we benchmark to the findings in Section 5.3 based on Svensson (1995) yields. We generally find that the estimated coefficients are adversely affected by using the more parsimonious specification of Nelson and Siegel (1987) to extract the synthetic yields. Most notably, the biases for $\kappa_{22}^{P}, \sigma_{11}, \sigma_{22}, \sigma_{33}$, and $\lambda$ increase somewhat when using the Nelson and Siegel (1987) yields compared to the Svensson (1995) yields, whereas the opposite applies for $\kappa_{11}^{P}$. Table 16 further shows, that the filtered state estimates with the Nelson and Siegel (1987) yields are less efficient (as measured by MAE) compared to the Svensson (1995) yields, whereas the mean errors are more similar across the two specifications.

Thus, restricting the parametric discount function for extracting synthetic zero-coupon yields compared to the specification in Svensson (1995) does not improve the performance of the conventional two-step approach. In terms of practical relevance, this finding provides support for the widely used synthetic U.S. Treasury yields constructed by Gürkaynak et al. $(2007,2010)$.

\subsection{Summarizing the Main Insights From the Monte Carlo Study}

The main insight from this Monte Carlo study is that the considered DTSM may be estimated more reliably by using directly observed market prices on coupon bonds instead of synthetic zero-coupon yields. Although these synthetic zero-coupon yields are estimated very 


\begin{tabular}{c|c|c|c|c||c|c|c|c}
\hline \hline \multirow{2}{*}{$\begin{array}{c}\text { State } \\
\text { variable }\end{array}$} & \multicolumn{4}{|c||}{ Svensson (1995) yields } & \multicolumn{4}{c}{ Nelson and Siegel (1987) yields } \\
\cline { 2 - 8 } & $\sigma_{\varepsilon}=1$ basis point & $\sigma_{\varepsilon}=10$ basis points & $\sigma_{\varepsilon}=1$ basis point & $\sigma_{\varepsilon}=10$ basis points \\
\cline { 2 - 8 } & Mean & MAE & Mean & MAE & Mean & MAE & Mean & MAE \\
\hline$L_{t}$ & -8.93 & 10.80 & -3.05 & 9.66 & 6.69 & 39.44 & -10.69 & 22.45 \\
$S_{t}$ & 14.28 & 15.67 & 7.45 & 13.46 & -2.78 & 37.30 & 15.13 & 26.69 \\
$C_{t}$ & -21.70 & 32.33 & -25.06 & 42.62 & -43.72 & 74.43 & -24.83 & 56.51 \\
\hline \hline
\end{tabular}

Table 16: Accuracy of Estimated States: Different Synthetic Yields

The table reports the mean of the sampling distribution of the mean errors (Mean) and mean absolute errors (MAE) of each estimated state variable in the AFNS model using ML in the two-step approach based on Svensson (1995) and Nelson and Siegel (1987) yields, each with the same ten maturities, 0.25, $0.5,1,2,3,5,7,10,20$, and 30 years. The mean is obtained by first computing the mean errors in each of the simulated samples across the $T=196$ observations, and we then report the average of these means across the $N=100$ simulated samples. Similarly, the MAE are obtained by first computing the mean absolute errors in each of the simulated samples across the $T=196$ observations, and we then report the average of these absolute means across the $N=100$ simulated samples. The true states are generated from the AFNS model as described in Section 5.1. All numbers are reported in basis points.

accurately with well-established curve-fitting techniques, we nevertheless find that seemingly negligible errors in these synthetic yields do affect the estimated parameters in DTSMs. In particular, all risk-neutral parameters are estimated with smaller biases and greater efficiency in the proposed one-step approach compared with the conventional two-step approach. This improvement is partly explained by the more densely representation of the yield curve in the one-step approach through the entire panel of coupon bond prices, and partly by the omission of seemingly negligible estimation errors in the synthetic zero-coupon yields. We also find that parameters in the $P$-dynamics benefit from a one-step approach, although these parameters are unrelated to the $Q$-dynamics with an essential affine specification for the market prices of risk. This improvement therefore arises mainly because the states are estimated with lower biases and greater precision in the one-step approach compared to the two-step approach. ${ }^{25}$

\section{Forecasting the Canadian Three-Month Yield}

The previous analysis has shown that parameters and latent states in the AFNS model are estimated with smaller biases and greater effeciency by the one-step approach when compared to the conventional two-step approach. The present section explores whether these advantages are sufficiently large to improve the ability of the AFNS model to forecast short-term bond yields out of sample.

We structure the forecast exercise to match the timing and frequency of the Consensus Forecasts, which serves as our benchmark, given that it is the survey for the Canadian econ-

\footnotetext{
${ }^{25}$ One potential minor caveat with our current Monte Carlo study is its relatively small number of simulations $(N=100)$. Ongoing work explores the robustness of our findings when $N$ is increased to 1,000 .
} 


\begin{tabular}{lccc}
\hline \hline Model & Mean & RMSE & MAE \\
\hline Consensus Forecasts & -84.97 & 122.25 & 85.07 \\
AFNS model: & & & \\
One-step approach & & & \\
All coupon bond prices & & & \\
Two-step approach & -79.52 & 115.80 & 89.08 \\
Bank of Canada yields, standard sample & -91.75 & 126.53 & 96.11 \\
Bank of Canada yields, extended sample & -100.98 & 130.03 & 101.60 \\
Bank of Canada yields, reduced sample & -80.91 & 120.09 & 87.76 \\
& & & \\
Svensson (1995) yields, standard sample & -83.10 & 115.90 & 88.01 \\
Svensson (1995) yields, extended sample & -98.76 & 128.47 & 100.52 \\
Svensson (1995) yields, reduced sample & -81.94 & 118.96 & 86.62 \\
\hline \hline
\end{tabular}

Table 17: Summary Statistics of Forecast Errors

All forecasts are created via independent-factor models with diagonal $K^{P}$. All numbers are measured in basis points.

omy with the longest history and largest panel of professional forecasters. To get a reasonable handle on the persistence of the states in the AFNS model, we begin the forecast analysis in December 2006. At the start of each month, the participants of the Consensus Forecasts submit their projections no later than the date indicated on the front page of the monthly release of the survey. Since we estimate our model with end-of-month data, we generate the corresponding model forecasts from the estimated model dynamics and filtered states for the month before the survey release. In this way, the model is handicapped by about a week relative to the survey panel. We adopt this cautious approach to ensure that the AFNS model does not use recent end-of-month information, which may have been unavailable to participants in the Consensus Forecasts. This implies that the reported results are conservative in terms of evaluating the forecasting ability of the AFNS model relative to the survey panel. Accordingly, the AFNS model is estimated in real time by adding one month of data to the sample at the time from December 2006 to April 2016, producing a total of 113 monthly estimations and forecasts.

Table 17 summarizes the outcome of this forecasting exercise for the Canadian threemonth yield. The Consensus Forecasts overpredict the level of this yield during our forecasting period, given its negative mean errors of -84.97 basis points. We see the same tendency in the forecasts from the AFNS model, where the use of the one-step approach to estimate this model gives the smallest (numerically) mean errors of -79.52 basis points. This version of the AFNS model is also seen to outperform the Consensus Forecasts in terms of root mean 
square errors (RMSE), which are 115.80 basis points for the AFNS model but 122.25 basis

points for Consensus Forecasts. However, when using the mean absolute errors (MAE), we obtain the opposite ranking. In line with our simulation study in Section 5, we also find that a reduced sample with only six synthetic yields in the two-step approach does better than using an extended sample with 31 synthetic yields.

Accordingly, the proposed one-step approach can also be used to generate competitive out of sample forecasts when compared to surveys and the conventional two-step approach with a limited number of yields.

\section{Conclusion}

This paper demonstrates the advantages of estimating DTSMs directly on actual bond prices as opposed to using a limited number of synthetic zero-coupon yields. For our Canadian sample of 105 bonds, we find that the risk-neutral parameters are those which are mostly affected by using this one-step approach, and that seemingly small differences between two data sets of synthetic yields with identical maturities can affect the estimated parameters in DTSMs. We also find that estimation of a DTSM by the one-step approach gives a substantially closer fit to actual bond prices than implied by the same model when it is estimated by the conventional two-step approach. This shows that the use of synthetic yields in the two-step approach may add a considerable amount of noise to the predicted bond prices from an estimated DTSM.

We also explore the finite-sample properties of the proposed one-step approach and the conventional two-step approach in a Monte Carlo study. A novel feature of this simulation experiment is to work at the level of coupon bonds to account for estimation uncertainty in the construction of synthetic zero-coupon yields. The main insight from this Monte Carlo study is that the risk-neutral parameters in DTSMs are estimated with smaller biases and greater efficiency in the one-step approach when compared to the conventional two-step approach.

There are obviously other advantages to estimating DTSMs directly on bond prices than what is covered in the present paper. For example, DTSMs could be augmented with bondspecific characteristics to enhance our understanding of liquidity premiums and their dynamics as in Fontaine and Garcia (2012) and Andreasen et al. (2017). Similarly, the proposed onestep approach reveals directly which bonds trade cheap or at a premium relative to the overall market, and this could be useful for portfolio management, arbitrage trading, and market surveillance. We leave these and other applications for future research. 


\section{Appendix A: Characteristics of Canadian Government Bonds}

Tables 18 to 20 provide the contractual characteristics of all 105 bonds and the number of monthly observations for each bond. Our sample includes all fixed-coupon bonds issued between January 2000 and April 2016 as well as 13 earlier bonds issued from 1990 to 1999. Generally, since 2008, each bond has had three or more auctions and its final total amount outstanding has been CAD 9 billion or more, which represents a substantial amount of notional.

\begin{tabular}{|c|c|c|c|c|c|}
\hline \multirow{2}{*}{ Bond: coupon, maturity } & \multirow{2}{*}{$\begin{array}{l}\text { No. } \\
\text { obs. }\end{array}$} & \multicolumn{2}{|c|}{ Issuance } & \multirow{2}{*}{$\begin{array}{c}\text { Number of } \\
\text { auctions }\end{array}$} & \multirow{2}{*}{$\begin{array}{c}\text { Total notional } \\
\text { amount }\end{array}$} \\
\hline & & Date & Amount & & \\
\hline (1) $10.5 \% 3 / 15 / 2021^{+}$ & 196 & $12 / 15 / 1990$ & n.a. & n.a. & 567 \\
\hline (2) $9.75 \% 6 / 1 / 2021^{+}$ & 196 & $5 / 9 / 1991$ & n.a. & n.a. & 286 \\
\hline (3) $9.25 \% 6 / 1 / 2022^{+}$ & 196 & $12 / 15 / 1991$ & n.a. & n.a. & 206 \\
\hline (4) $8 \% 6 / 1 / 2023^{+}$ & 196 & 8/17/1992 & n.a. & n.a. & 2,358 \\
\hline (5) $9 \% 6 / 1 / 2025^{+}$ & 196 & $8 / 2 / 1994$ & n.a. & n.a. & 2,303 \\
\hline (6) $8 \% 6 / 1 / 2027^{+}$ & 196 & $5 / 1 / 1996$ & n.a. & n.a. & 4,036 \\
\hline (7) $5.75 \% 6 / 1 / 2029^{+}$ & 196 & $2 / 2 / 1998$ & n.a. & n.a. & 10,950 \\
\hline (8) $4.5 \% 6 / 1 / 2001$ & 14 & $12 / 15 / 1998$ & 3,500 & 2 & 7,000 \\
\hline (9) $5 \% 9 / 1 / 2004^{*}$ & 53 & 3/1/1999 & 2,500 & 4 & 10,850 \\
\hline (10) $5.25 \% \quad 12 / 1 / 2001$ & 20 & $6 / 15 / 1999$ & 3,500 & 2 & 7,000 \\
\hline (11) $5.5 \% 6 / 1 / 2010^{\dagger}$ & 122 & 8/3/1999 & 2,600 & 4 & 10,400 \\
\hline (12) $6 \% 9 / 1 / 2005^{*}$ & 65 & $11 / 15 / 1999$ & 2,800 & 4 & 11,100 \\
\hline (13) $5.75 \% 6 / 1 / 2002$ & 26 & $12 / 1 / 1999$ & 3,600 & 2 & 7,200 \\
\hline (14) $6 \% 6 / 1 / 2011^{\dagger}$ & 131 & $5 / 1 / 2000$ & 2,600 & 6 & 15,000 \\
\hline (15) $6 \% 12 / 1 / 2002$ & 27 & $6 / 15 / 2000$ & 3,600 & 2 & 7,100 \\
\hline (16) $5.75 \% 9 / 1 / 2006^{*}$ & 67 & $11 / 14 / 2000$ & 2,500 & 4 & 10,000 \\
\hline (17) $5.75 \% 6 / 1 / 2003$ & 28 & $11 / 24 / 2000$ & 3,500 & 2 & 7,000 \\
\hline (18) $5 \%$ 12/1/2003 & 27 & $6 / 15 / 2001$ & 3,500 & 2 & 7,000 \\
\hline (19) $5.75 \% 6 / 1 / 2033^{+}$ & 175 & $10 / 15 / 2001$ & 2,000 & 6 & 10,700 \\
\hline (20) $5.25 \% 6 / 1 / 2012^{\dagger}$ & 125 & $10 / 29 / 2001$ & 2,500 & 4 & 9,900 \\
\hline (21) $4.5 \% 9 / 1 / 2007^{*}$ & 67 & $11 / 19 / 2001$ & 2,500 & 4 & 9,800 \\
\hline (22) $3.5 \% 6 / 1 / 2004$ & 28 & $11 / 30 / 2001$ & 3,500 & 2 & 7,000 \\
\hline (23) $4.25 \% \quad 12 / 1 / 2004$ & 28 & $5 / 31 / 2002$ & 3,500 & 2 & 6,500 \\
\hline (24) $5.25 \% 6 / 1 / 2013^{\dagger}$ & 125 & $11 / 4 / 2002$ & 2,400 & 4 & 9,600 \\
\hline (25) $4.25 \% 9 / 1 / 2008^{*}$ & 67 & $11 / 18 / 2002$ & 2,400 & 4 & 9,400 \\
\hline (26) $3.5 \% 6 / 1 / 2005$ & 28 & $11 / 29 / 2002$ & 3,500 & 2 & 7,000 \\
\hline (27) $3 \% 12 / 1 / 2005$ & 27 & $6 / 13 / 2003$ & 2,700 & 2 & 5,200 \\
\hline (28) $5 \% 6 / 1 / 2014^{\dagger}$ & 125 & $10 / 20 / 2003$ & 2,400 & 4 & 9,100 \\
\hline (29) $4.25 \% 9 / 1 / 2009$ & 67 & $12 / 1 / 2003$ & 2,300 & 4 & 8,800 \\
\hline (30) $3 \% 6 / 1 / 2006$ & 27 & $12 / 19 / 2003$ & 3,500 & 2 & 7,000 \\
\hline (31) $3.25 \% \quad 12 / 1 / 2006$ & 28 & $5 / 28 / 2004$ & 2,800 & 2 & 5,600 \\
\hline (32) $5 \% 6 / 1 / 2037^{+}$ & 142 & $7 / 19 / 2004$ & 1,500 & 8 & 11,000 \\
\hline (33) $4.5 \% 6 / 1 / 2015^{\dagger}$ & 125 & $10 / 18 / 2004$ & 2,100 & 4 & 8,400 \\
\hline (34) $4 \% 9 / 1 / 2010^{*}$ & 67 & $11 / 22 / 2004$ & 2,100 & 4 & 8,100 \\
\hline (35) $3 \% 6 / 1 / 2007$ & 27 & $12 / 10 / 2004$ & 2,800 & 2 & 5,300 \\
\hline
\end{tabular}

Table 18: Sample of Canadian Government Fixed-Coupon Bonds

The table reports the characteristics, first issuance date and amount, the total number of auctions, and total amount issued in millions of Canadian dollars for the Canadian government fixed-coupon bonds in the sample. Also reported are the number of monthly observation dates for each bond during the sample period from January 31, 2000, to April 29, 2016. Asterisk * indicates five-year bonds, dagger $\dagger$ indicates ten-year bonds, plus + indicates thirty-year bonds, and cross $\times$ indicates fifty-year bonds based on the official maturity grouping used by the Bank of Canada. 


\begin{tabular}{|c|c|c|c|c|c|}
\hline \multirow{2}{*}{ Bond: coupon, maturity } & \multirow{2}{*}{$\begin{array}{l}\text { No. } \\
\text { obs. }\end{array}$} & \multicolumn{2}{|c|}{ Issuance } & \multirow{2}{*}{$\begin{array}{c}\text { Number of } \\
\text { auctions }\end{array}$} & \multirow{2}{*}{$\begin{array}{c}\text { Total notional } \\
\text { amount }\end{array}$} \\
\hline & & Date & Amount & & \\
\hline (36) $2.75 \% \quad 12 / 1 / 2007$ & 27 & $6 / 10 / 2005$ & 3,400 & 2 & 6,800 \\
\hline (37) $4 \% 6 / 1 / 2016^{\dagger}$ & 124 & $11 / 7 / 2005$ & 2,100 & 4 & 8,700 \\
\hline (38) $3.75 \% 9 / 1 / 2011^{*}$ & 67 & $11 / 21 / 2005$ & 1,900 & 4 & 8,000 \\
\hline (39) $3.75 \% 6 / 1 / 2008$ & 27 & $12 / 16 / 2005$ & 2,400 & 1 & 2,400 \\
\hline (40) $4.25 \% \quad 12 / 1 / 2008$ & 27 & $6 / 16 / 2006$ & 3,400 & 2 & 6,800 \\
\hline (41) $4 \% 6 / 1 / 2017^{\dagger}$ & 115 & $10 / 16 / 2006$ & 2,300 & 4 & 9,800 \\
\hline (42) $3.75 \% 6 / 1 / 2012^{*}$ & 64 & $11 / 6 / 2006$ & 2,000 & 3 & 6,000 \\
\hline (43) $3.75 \%$ 6/1/2009 & 28 & $11 / 17 / 2006$ & 2,400 & 1 & 2,400 \\
\hline (44) $4.25 \% \quad 12 / 1 / 2009$ & 28 & $5 / 25 / 2007$ & 3,500 & 2 & 7,100 \\
\hline (45) $4.25 \% 6 / 1 / 2018^{\dagger}$ & 103 & $10 / 29 / 2007$ & 2,500 & 5 & 10,100 \\
\hline (46) $3.75 \% 6 / 1 / 2010$ & 28 & $11 / 30 / 2007$ & 3,300 & 1 & 3,300 \\
\hline (47) $3.5 \% 6 / 1 / 2013^{*}$ & 61 & $2 / 25 / 2008$ & 2,000 & 5 & 15,000 \\
\hline (48) $2.75 \% \quad 12 / 1 / 2010$ & 28 & $5 / 23 / 2008$ & 3,500 & 3 & 11,600 \\
\hline (49) $4 \% 6 / 1 / 2041^{+}$ & 95 & $6 / 9 / 2008$ & 1,400 & 10 & 14,100 \\
\hline (50) $3.75 \% 6 / 1 / 2019^{\dagger}$ & 91 & $10 / 6 / 2008$ & 2,500 & 5 & 16,000 \\
\hline (51) $3 \% 6 / 1 / 2014^{*}$ & 65 & $10 / 21 / 2008$ & 3,000 & 4 & 16,000 \\
\hline (52) $1.25 \% 6 / 1 / 2011$ & 26 & $1 / 27 / 2009$ & 4,500 & 3 & 11,000 \\
\hline (53) $2 \% 12 / 1 / 2014^{*}$ & 65 & $4 / 20 / 2009$ & 3,000 & 5 & 15,000 \\
\hline (54) $1 \% 9 / 1 / 2011$ & 25 & $5 / 8 / 2009$ & 3,500 & 3 & 10,000 \\
\hline (55) $2 \% 9 / 1 / 2012$ & 37 & $6 / 1 / 2009$ & 3,500 & 5 & 16,500 \\
\hline (56) $1.25 \% \quad 12 / 1 / 2011$ & 25 & $8 / 21 / 2009$ & 3,000 & 3 & 9,500 \\
\hline (57) $3.5 \% 6 / 1 / 2020^{\dagger}$ & 80 & $9 / 8 / 2009$ & 3,000 & 4 & 12,500 \\
\hline (58) $1.5 \% 3 / 1 / 2012$ & 25 & $11 / 16 / 2009$ & 3,000 & 3 & 9,000 \\
\hline (59) $2.5 \% 6 / 1 / 2015^{*}$ & 64 & $11 / 23 / 2009$ & 3,000 & 3 & 9,000 \\
\hline (60) $1.75 \% 3 / 1 / 2013$ & 35 & $12 / 14 / 2009$ & 3,200 & 6 & 18,600 \\
\hline (61) $1.5 \% 6 / 1 / 2012$ & 24 & $3 / 12 / 2010$ & 3,000 & 3 & 9,000 \\
\hline (62) $3 \% 12 / 1 / 2015^{*}$ & 65 & $4 / 19 / 2010$ & 3,500 & 3 & 10,500 \\
\hline (63) $2.5 \% 9 / 1 / 2013$ & 37 & $5 / 17 / 2010$ & 3,000 & 3 & 9,200 \\
\hline (64) $3.25 \% 6 / 1 / 2021^{\dagger}$ & 70 & $7 / 19 / 2010$ & 3,000 & 4 & 11,500 \\
\hline $\begin{array}{lll} & 1.5 \% & 12 / 1 / 2012\end{array}$ & 25 & $8 / 13 / 2010$ & 3,000 & 4 & 12,000 \\
\hline (66) $2 \% 6 / 1 / 2016^{*}$ & 64 & $11 / 8 / 2010$ & 3,500 & 3 & 9,900 \\
\hline (67) $2 \% 3 / 1 / 2014$ & 36 & $12 / 13 / 2010$ & 3,200 & 3 & 9,600 \\
\hline (68) $2 \% 8 / 1 / 2013$ & 25 & $4 / 8 / 2011$ & 3,500 & 3 & 10,500 \\
\hline (69) $2.75 \% 9 / 1 / 2016^{*}$ & 61 & $4 / 26 / 2011$ & 3,500 & 3 & 10,500 \\
\hline (70) $2.25 \% 8 / 1 / 2014$ & 37 & $5 / 2 / 2011$ & 3,000 & 5 & 15,600 \\
\hline
\end{tabular}

Table 19: Sample of Canadian Government Fixed-Coupon Bonds cont.

The table reports the characteristics, first issuance date and amount, the total number of auctions, and total amount issued in millions of Canadian dollars for the Canadian government fixed-coupon bonds in the sample. Also reported are the number of monthly observation dates for each bond during the sample period from January 31, 2000, to April 29, 2016. Asterisk * indicates five-year bonds, dagger $\dagger$ indicates ten-year bonds, plus + indicates thirty-year bonds, and cross $\times$ indicates fifty-year bonds based on the official maturity grouping used by the Bank of Canada. 


\begin{tabular}{|c|c|c|c|c|c|}
\hline \multirow{2}{*}{ Bond: coupon, maturity } & \multirow{2}{*}{$\begin{array}{l}\text { No. } \\
\text { obs. }\end{array}$} & \multicolumn{2}{|c|}{ Issuance } & \multirow{2}{*}{$\begin{array}{c}\text { Number of } \\
\text { auctions }\end{array}$} & \multirow{2}{*}{$\begin{array}{c}\text { Total notional } \\
\text { amount }\end{array}$} \\
\hline & & Date & Amount & & \\
\hline (71) $3.5 \% 12 / 1 / 2045^{+}$ & 59 & $6 / 13 / 2011$ & 1,400 & 10 & 14,400 \\
\hline (72) $1.5 \% 11 / 1 / 2013$ & 25 & $7 / 15 / 2011$ & 3,500 & 3 & 10,500 \\
\hline (73) $2.75 \% 6 / 1 / 2022^{\dagger}$ & 58 & $8 / 2 / 2011$ & 2,500 & 5 & 12,700 \\
\hline (74) $1.5 \% 3 / 1 / 2017^{*}$ & 55 & $10 / 17 / 2011$ & 3,500 & 3 & 10,500 \\
\hline (75) $1 \% 2 / 1 / 2014$ & 25 & $10 / 21 / 2011$ & 3,500 & 3 & 10,500 \\
\hline (76) $1 \% 2 / 1 / 2015$ & 36 & $11 / 7 / 2011$ & 3,000 & 5 & 15,600 \\
\hline (77) $0.75 \% 5 / 1 / 2014$ & 24 & $1 / 13 / 2012$ & 3,500 & 3 & 10,500 \\
\hline (78) $1.5 \% 8 / 1 / 2015$ & 37 & $4 / 30 / 2012$ & 2,900 & 5 & 15,300 \\
\hline (79) $1.5 \% 8 / 1 / 2017^{*}$ & 48 & $5 / 14 / 2012$ & 3,400 & 3 & 10,200 \\
\hline (80) $1 \% 11 / 1 / 2014$ & 26 & $6 / 22 / 2012$ & 3,300 & 3 & 9,900 \\
\hline (81) $1.5 \% 6 / 1 / 2023^{\dagger}$ & 46 & $7 / 30 / 2012$ & 2,600 & 5 & 14,200 \\
\hline (82) $1.25 \% 2 / 1 / 2016$ & 37 & $10 / 15 / 2012$ & 2,700 & 5 & 14,700 \\
\hline (83) $1.25 \% 3 / 1 / 2018^{*}$ & 42 & $11 / 13 / 2012$ & 3,400 & 3 & 10,200 \\
\hline (84) $1 \% 5 / 1 / 2015$ & 24 & $1 / 18 / 2013$ & 3,300 & 3 & 9,900 \\
\hline (85) $1 \% 8 / 1 / 2016$ & 37 & $4 / 15 / 2013$ & 2,700 & 6 & 17,100 \\
\hline (86) $1.25 \% 9 / 1 / 2018^{*}$ & 36 & $5 / 13 / 2013$ & 3,400 & 3 & 10,200 \\
\hline (87) $2.5 \% 6 / 1 / 2024^{\dagger}$ & 35 & $7 / 2 / 2013$ & 2,800 & 5 & 13,800 \\
\hline (88) $1 \% \quad 11 / 1 / 2015$ & 25 & $7 / 26 / 2013$ & 3,300 & 3 & 9,900 \\
\hline (89) $1.5 \% 2 / 1 / 2017$ & 31 & $10 / 15 / 2013$ & 2,700 & 6 & 17,100 \\
\hline (90) $1.75 \% 3 / 1 / 2019^{*}$ & 30 & $11 / 12 / 2013$ & 3,400 & 3 & 10,200 \\
\hline (91) $1 \% 5 / 1 / 2016$ & 25 & $1 / 31 / 2014$ & 3,300 & 3 & 10,000 \\
\hline (92) $1.75 \% 9 / 1 / 2019^{*}$ & 25 & $4 / 14 / 2014$ & 3,400 & 3 & 10,200 \\
\hline (93) $2.75 \% 12 / 1 / 2064^{\times}$ & 25 & $4 / 28 / 2014$ & n.a. & n.a. & 3,500 \\
\hline (94) $1.25 \% 8 / 1 / 2017$ & 24 & $5 / 20 / 2014$ & 2,700 & 6 & 19,100 \\
\hline (95) $2.75 \% 12 / 1 / 2048^{+}$ & 24 & $6 / 2 / 2014$ & 1,400 & 5 & 7,000 \\
\hline (96) $2.25 \% 6 / 1 / 2025^{\dagger}$ & 23 & $6 / 30 / 2014$ & 2,700 & 5 & 13,100 \\
\hline (97) $1 \% 11 / 1 / 2016$ & 21 & $8 / 15 / 2014$ & 3,400 & 3 & 10,200 \\
\hline (98) $1.5 \% 3 / 1 / 2020^{*}$ & 19 & $10 / 14 / 2014$ & 3,400 & 3 & 10,200 \\
\hline (99) $1.25 \% 2 / 1 / 2018$ & 18 & $11 / 10 / 2014$ & 2,700 & 6 & 19,200 \\
\hline (100) $0.25 \% 5 / 1 / 2017$ & 15 & $2 / 13 / 2015$ & 3,400 & 3 & 10,400 \\
\hline (101) $0.75 \% 9 / 1 / 2020$ & 13 & $4 / 13 / 2015$ & 3,300 & 4 & 13,000 \\
\hline (102) $1.5 \% 6 / 1 / 2026^{\dagger}$ & 10 & $7 / 21 / 2015$ & 2,500 & 4 & 10,500 \\
\hline (103) $0.25 \% \quad 11 / 1 / 2017$ & 9 & $8 / 7 / 2015$ & 3,300 & 4 & 13,400 \\
\hline (104) $0.75 \% 3 / 1 / 2021^{*}$ & 7 & $10 / 19 / 2015$ & 3,300 & 4 & 13,800 \\
\hline (105) $0.25 \% 5 / 1 / 2018$ & 3 & $2 / 5 / 2016$ & 3,700 & 3 & 11,100 \\
\hline
\end{tabular}

Table 20: Sample of Canadian Government Fixed-Coupon Bonds cont.

The table reports the characteristics, first issuance date and amount, the total number of auctions, and total amount issued in millions of Canadian dollars for the Canadian government fixed-coupon bonds in the sample. Also reported are the number of monthly observation dates for each bond during the sample period from January 31, 2000, to April 29, 2016. Asterisk * indicates five-year bonds, dagger $\dagger$ indicates ten-year bonds, plus + indicates thirty-year bonds, and cross $\times$ indicates fifty-year bonds based on the official maturity grouping used by the Bank of Canada. 


\section{Appendix B: Construction of Zero-Coupon Yields}

This section describes how we construct zero-coupon yields by the Svensson (1995) discount function using the panel of Canadian government fixed-coupon bond prices analyzed in the main text. The Svensson (1995) yield curve has a flexible functional form given by

$$
y_{t}(\tau)=\beta_{0}(t)+\frac{1-e^{-\lambda_{1} \tau}}{\lambda_{1} \tau} \beta_{1}(t)+\left(\frac{1-e^{-\lambda_{1} \tau}}{\lambda_{1} \tau}-e^{-\lambda_{1} \tau}\right) \beta_{2}(t)+\left(\frac{1-e^{-\lambda_{2} \tau}}{\lambda_{2} \tau}-e^{-\lambda_{2} \tau}\right) \beta_{3}(t),
$$

where we impose the restrictions that $\lambda_{1}>\lambda_{2}>0$. This function contains the level, slope, and curvature components known from Nelson and Siegel (1987) and augments them with an additional curvature factor to provide a better fit to the long end of the yield curve. The corresponding discount function is easily obtained as $P_{t}^{\mathrm{zc}}(\tau)=e^{-y_{t}(\tau) \tau}$. Now, consider the value at time $t$ of a fixed-coupon bond with maturity at $t+\tau$ that pays an annual coupon $C$ semi-annually. Its price, denoted $P_{t}(\tau, C)$, is simply the sum of its remaining cash flow payments weighted by the zero-coupon bond price function $P_{t}^{\mathrm{zc}}(\tau)$ :

$$
P_{t}(\tau, C)=\frac{C}{2} \frac{\left(t_{1}-t\right)}{1 / 2} P_{t}^{\mathrm{zc}}\left(t_{1}\right)+\sum_{j=2}^{N} \frac{C}{2} P_{t}^{\mathrm{zc}}\left(t_{j}\right)+P_{t}^{\mathrm{zc}}(\tau), \quad t<t_{1}<\ldots<t_{N}=\tau .
$$

For each observation date, the parameters in the Svensson (1995) curve, $\left(\beta_{0}, \beta_{1}, \beta_{2}, \beta_{3}, \lambda_{1}, \lambda_{2}\right)$, are estimated by optimizing the following objective function

$$
\min \sum_{i=1}^{n_{y, t}} \frac{1}{D_{t}^{i}}\left(P_{t}^{\text {data,i }}-\widehat{P_{t}^{i}}\right)^{2}
$$

where $n_{y, t}$ is the number of coupon bond prices observed on day $t, P_{t}^{\text {data,i }}$ is the observed price for bond number $i, \widehat{P_{t}^{i}}$ is its price implied by the Svensson (1995) discount function, and $D_{t}^{i}$ is its duration, which is model-free and calculated before estimation based on the Macaulay formula. The stated objective is to minimize the weighted sum of the squared deviations between the actual bond prices and the predicted prices, where the weights are the inverse of the durations of each individual security. This is identical to the objective function used by Gürkaynak et al. (2007, 2010). The optimization for each observation date is started at the same parameter vector:

$$
\left(\begin{array}{l}
\beta_{0} \\
\beta_{1} \\
\beta_{2} \\
\beta_{3} \\
\lambda_{1} \\
\lambda_{2}
\end{array}\right)=\left(\begin{array}{c}
0.04173257 \\
-0.02703468 \\
-0.05262533 \\
0.02954742 \\
0.8378759 \\
0.09652915
\end{array}\right)
$$

Tables 21 and 22 report the summary statistics of the mean errors and the mean absolute errors for the ten constant-maturity zero-coupon yields constructed using the Svensson (1995) yield curve as described above when the underlying bond prices are simulated from the AFNS model as described in Section 5.1 with measurement error standard deviation equal to $\sigma_{\varepsilon}=1$ basis point and $\sigma_{\varepsilon}=10$ basis points, respectively. 


\begin{tabular}{c|c|c||c|c|c|c|c}
\hline \hline \multirow{2}{*}{$\begin{array}{c}\text { Maturity } \\
\text { in months }\end{array}$} & \multicolumn{7}{|c}{ Mean errors, $\sigma_{\varepsilon}=1$ basis point } \\
\cline { 2 - 7 } & Mean & Std. dev. & 5 percentile & $1^{\text {st }}$ quartile & Median & $3^{\text {rd }}$ quartile & 95 percentile \\
\hline 3 & -4.09 & 2.83 & -9.48 & -5.70 & -3.75 & -2.16 & 0.09 \\
6 & -2.37 & 1.69 & -5.78 & -3.34 & -1.99 & -1.21 & -0.18 \\
12 & -0.32 & 0.42 & -1.19 & -0.61 & -0.26 & 0.01 & 0.20 \\
24 & 0.78 & 0.60 & -0.17 & 0.39 & 0.69 & 1.07 & 1.82 \\
36 & 0.43 & 0.47 & -0.10 & 0.07 & 0.30 & 0.73 & 1.32 \\
60 & -0.48 & 0.37 & -1.11 & -0.68 & -0.45 & -0.25 & 0.05 \\
84 & -0.67 & 0.62 & -1.94 & -1.00 & -0.51 & -0.21 & 0.07 \\
120 & -0.34 & 0.48 & -1.06 & -0.71 & -0.27 & 0.05 & 0.31 \\
240 & 0.64 & 0.68 & -0.36 & 0.07 & 0.57 & 1.02 & 1.91 \\
360 & -1.32 & 1.14 & -3.12 & -2.23 & -1.36 & -0.49 & 0.41 \\
\hline \hline
\end{tabular}

\begin{tabular}{c|c|c||c|c|c|c|c}
\hline \hline \multirow{2}{*}{$\begin{array}{c}\text { Maturity } \\
\text { in months }\end{array}$} & \multicolumn{7}{|c}{ Mean absolute errors, $\sigma_{\varepsilon}=1$ basis point } \\
\cline { 2 - 8 } & Mean & Std. dev. & 5 percentile & $1^{\text {st }}$ quartile & Median & $3^{\text {rd }}$ quartile & 95 percentile \\
\hline 3 & 6.79 & 2.55 & 4.00 & 4.80 & 6.15 & 7.84 & 11.57 \\
6 & 3.89 & 1.63 & 2.19 & 2.64 & 3.42 & 4.65 & 6.99 \\
12 & 1.05 & 0.40 & 0.57 & 0.76 & 0.95 & 1.28 & 1.83 \\
24 & 1.42 & 0.51 & 0.87 & 1.03 & 1.27 & 1.64 & 2.35 \\
36 & 0.99 & 0.45 & 0.49 & 0.66 & 0.84 & 1.23 & 1.84 \\
60 & 1.05 & 0.25 & 0.74 & 0.87 & 1.01 & 1.14 & 1.49 \\
84 & 1.26 & 0.56 & 0.62 & 0.81 & 1.08 & 1.56 & 2.34 \\
120 & 0.94 & 0.28 & 0.61 & 0.73 & 0.86 & 1.11 & 1.43 \\
240 & 1.46 & 0.53 & 0.85 & 1.04 & 1.38 & 1.72 & 2.64 \\
360 & 3.09 & 0.81 & 1.93 & 2.40 & 2.97 & 3.62 & 4.62 \\
\hline \hline
\end{tabular}

\section{Table 21: Summary Statistics of Errors of Constructed Yields}

The top panel reports the summary statistics of the mean errors between the true zero-coupon bond yields and the zero-coupon bond yields constructed using the Svensson (1995) yield curve based on $N=100$ simulated data sets generated by the independent-factor AFNS model, each with a distribution of coupon bond prices identical to the sample of Canadian fixed-coupon bond prices described in Section 2 and with each simulated bond price being added an i.i.d. measurement error with zero mean and a uniform measurement error standard deviation of $\sigma_{\varepsilon}=1$ basis point scaled by the bond's duration. The bottom panel reports the corresponding summary statistics of the mean absolute errors. All numbers are measured in basis points. 


\begin{tabular}{c|c|c||c|c|c|c|c}
\hline \hline \multirow{2}{*}{$\begin{array}{c}\text { Maturity } \\
\text { in months }\end{array}$} & \multicolumn{7}{|c}{ Mean errors, $\sigma_{\varepsilon}=10$ basis points } \\
\cline { 2 - 7 } & Mean & Std. dev. & 5 percentile & $1^{\text {st }}$ quartile & Median & $3^{\text {rd }}$ quartile & 95 percentile \\
\hline 3 & -3.00 & 2.59 & -7.12 & -4.61 & -2.79 & -1.02 & 1.44 \\
6 & -1.74 & 1.54 & -4.37 & -2.72 & -1.66 & -0.58 & 0.71 \\
12 & -0.24 & 0.48 & -1.07 & -0.54 & -0.30 & 0.06 & 0.67 \\
24 & 0.52 & 0.57 & -0.52 & 0.22 & 0.46 & 0.85 & 1.43 \\
36 & 0.24 & 0.44 & -0.37 & -0.11 & 0.23 & 0.56 & 1.04 \\
60 & -0.34 & 0.45 & -1.16 & -0.63 & -0.26 & -0.03 & 0.25 \\
84 & -0.37 & 0.58 & -1.56 & -0.64 & -0.28 & 0.06 & 0.34 \\
120 & -0.14 & 0.43 & -0.85 & -0.52 & -0.04 & 0.18 & 0.43 \\
240 & 0.27 & 0.65 & -0.90 & -0.18 & 0.31 & 0.61 & 1.19 \\
360 & -0.90 & 1.31 & -3.07 & -1.78 & -1.16 & 0.13 & 1.16 \\
\hline \hline
\end{tabular}

\begin{tabular}{c|c|c||c|c|c|c|c}
\hline \hline \multirow{2}{*}{$\begin{array}{c}\text { Maturity } \\
\text { in months }\end{array}$} & \multicolumn{7}{|c}{ Mean absolute errors, $\sigma_{\varepsilon}=10$ basis points } \\
\cline { 2 - 8 } & Mean & Std. dev. & 5 percentile & $1^{\text {st }}$ quartile & Median & $3^{\text {rd }}$ quartile & 95 percentile \\
\hline 3 & 12.48 & 1.44 & 10.47 & 11.50 & 12.21 & 13.15 & 15.58 \\
6 & 7.84 & 0.91 & 6.67 & 7.24 & 7.62 & 8.33 & 9.34 \\
12 & 3.58 & 0.27 & 3.17 & 3.41 & 3.54 & 3.77 & 4.05 \\
24 & 3.52 & 0.26 & 3.09 & 3.36 & 3.52 & 3.69 & 3.93 \\
36 & 3.12 & 0.22 & 2.78 & 2.96 & 3.12 & 3.30 & 3.46 \\
60 & 3.13 & 0.22 & 2.80 & 2.97 & 3.14 & 3.28 & 3.50 \\
84 & 3.30 & 0.21 & 2.96 & 3.17 & 3.30 & 3.42 & 3.64 \\
120 & 2.88 & 0.21 & 2.48 & 2.75 & 2.85 & 3.00 & 3.19 \\
240 & 3.76 & 0.34 & 3.23 & 3.49 & 3.80 & 3.99 & 4.26 \\
360 & 9.34 & 1.28 & 7.58 & 8.48 & 9.12 & 10.13 & 11.53 \\
\hline \hline
\end{tabular}

Table 22: Summary Statistics of Errors of Constructed Yields

The top panel reports the summary statistics of the mean errors between the true zero-coupon bond yields and the zero-coupon bond yields constructed using the Svensson (1995) yield curve based on $N=100$ simulated data sets generated by the independent-factor AFNS model, each with a distribution of coupon bond prices identical to the sample of Canadian fixed-coupon bond prices described in Section 2 and with each simulated bond price being added an i.i.d. measurement error with zero mean and a uniform measurement error standard deviation of $\sigma_{\varepsilon}=10$ basis points scaled by the bond's duration. The bottom panel reports the corresponding summary statistics of the mean absolute errors. All numbers are measured in basis points. 


\section{Appendix C: The Extended Kalman Filter Estimation}

This appendix describes the model estimations based on the Kalman filter and the extended Kalman filter. For affine Gaussian models in general, the conditional mean vector and the conditional covariance matrix are ${ }^{26}$

$$
\begin{aligned}
E^{P}\left[X_{t+\Delta t} \mid \mathcal{F}_{t}\right] & =\left(I-\exp \left(-K^{P} \Delta t\right)\right) \theta^{P}+\exp \left(-K^{P} \Delta t\right) X_{t}, \\
V^{P}\left[X_{t+\Delta t} \mid \mathcal{F}_{t}\right] & =\int_{t}^{t+\Delta t} e^{-K^{P} s} \Sigma \Sigma^{\prime-\left(K^{P}\right)^{\prime} s} d s
\end{aligned}
$$

where $\Delta t$ is the time between observations. Conditional moments of discrete observations are computed and the state transition equation is obtained as

$$
X_{t}=\left(I-\exp \left(-K^{P} \Delta t\right)\right) \theta^{P}+\exp \left(-K^{P} \Delta t\right) X_{t-\Delta t}+\xi_{t},
$$

where $\xi_{t}$ refers to the Gaussian state innovations.

In the standard Kalman filter, the measurement equation is linear

$$
y_{t}=A+B X_{t}+\varepsilon_{t},
$$

and the assumed error structure is

$$
\left(\begin{array}{c}
\xi_{t} \\
\varepsilon_{t}
\end{array}\right) \sim N\left[\left(\begin{array}{l}
0 \\
0
\end{array}\right),\left(\begin{array}{cc}
Q & 0 \\
0 & H
\end{array}\right)\right],
$$

where the matrix $H$ is assumed to be diagonal, while the matrix $Q$ has the following structure

$$
Q=\int_{0}^{\Delta t} e^{-K^{P} s} \Sigma \Sigma^{\prime-\left(K^{P}\right)^{\prime} s} d s
$$

In addition, the transition and measurement errors are assumed to be orthogonal to the initial states. Due to the assumed stationarity, the Kalman filter is initialized at the unconditional mean and variance of the state variables under the $P$-measure, i.e., $X_{0}=\theta^{P}$ and $\Sigma_{0}=\int_{0}^{\infty} e^{-K^{P} s} \Sigma \Sigma^{\prime-\left(K^{P}\right)^{\prime} s} d s$. Denote the information available at time $t$ by $Y_{t}=\left(y_{1}, y_{2}, \ldots, y_{t}\right)$, and denote model parameters by $\psi$. Let $\Delta t=1$ and consider period $t-1$ and suppose that the state update $X_{t-1}$ and its mean square error matrix $\Sigma_{t-1}$ have been obtained. The prediction step is

$$
\begin{gathered}
X_{t \mid t-1}=E^{P}\left[X_{t} \mid Y_{t-1}\right]=\Phi_{t}^{X, 0}(\psi)+\Phi_{t}^{X, 1}(\psi) X_{t-1}, \\
\Sigma_{t \mid t-1}=\Phi_{t}^{X, 1}(\psi) \Sigma_{t-1} \Phi_{t}^{X, 1}(\psi)^{\prime}+Q_{t}(\psi),
\end{gathered}
$$

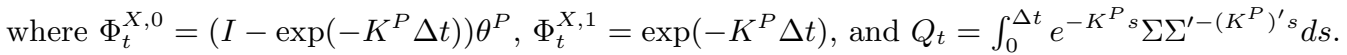

In the time- $t$ update step, $X_{t \mid t-1}$ is improved by using the additional information contained in $Y_{t}$, i.e.,

$$
\begin{gathered}
X_{t}=E\left[X_{t} \mid Y_{t}\right]=X_{t \mid t-1}+\Sigma_{t \mid t-1} B(\psi)^{\prime} F_{t}^{-1} v_{t}, \\
\Sigma_{t}=\Sigma_{t \mid t-1}-\Sigma_{t \mid t-1} B(\psi)^{\prime} F_{t}^{-1} B(\psi) \Sigma_{t \mid t-1},
\end{gathered}
$$

where

$$
\begin{gathered}
v_{t}=y_{t}-E\left[y_{t} \mid Y_{t-1}\right]=y_{t}-A(\psi)-B(\psi) X_{t \mid t-1}, \\
F_{t}=\operatorname{cov}\left(v_{t}\right)=B(\psi) \Sigma_{t \mid t-1} B(\psi)^{\prime}+H(\psi), \\
H(\psi)=\operatorname{diag}\left(\sigma_{\varepsilon}^{2}\left(\tau_{1}\right), \ldots, \sigma_{\varepsilon}^{2}\left(\tau_{N}\right)\right) .
\end{gathered}
$$

\footnotetext{
${ }^{26}$ Throughout, conditional and unconditional covariance matrices are calculated using the analytical solutions provided in Fisher and Gilles (1996).
} 
At this point, the Kalman filter has delivered all ingredients needed to evaluate the Gaussian log likelihood, which reads

$$
L^{E K F}(\psi) \equiv \log l\left(y_{1}, \ldots, y_{T} ; \psi\right)=\sum_{t=1}^{T}\left(-\frac{N}{2} \log (2 \pi)-\frac{1}{2} \log \left|F_{t}\right|-\frac{1}{2} v_{t}^{\prime} F_{t}^{-1} v_{t}\right)
$$

where $N$ is the number of observed yields. Now, the likelihood is numerically maximized with respect to $\psi$ using the Nelder-Mead simplex algorithm. Upon convergence, the standard errors are obtained from the estimated covariance matrix,

$$
\widehat{\Omega}(\widehat{\psi})=\frac{1}{T}\left[\frac{1}{T} \sum_{t=1}^{T} \frac{\partial \log l_{t}(\widehat{\psi})}{\partial \psi} \frac{\partial \log l_{t}(\widehat{\psi})^{\prime}}{\partial \psi}\right]^{-1}
$$

where $\widehat{\psi}$ denotes the estimated model parameters.

In model estimations with coupon bond prices in the one-step approach and for the B-AFNS model using the two-step approach, the extended Kalman filter is needed because the measurement equations are no longer affine functions of the states. Instead, the measurement equation takes the general form

$$
\frac{\bar{P}_{t}^{i}\left(t_{0}^{i}, \tau^{i}\right)}{D_{t}^{i}\left(t_{0}^{i}, \tau^{i}\right)}=g\left(X_{t} ; t_{0}^{i}, \tau^{i}, \psi\right)+\varepsilon_{t}^{i}
$$

In the extended Kalman filter, this equation is linearized using a first-order Taylor expansion around the best guess of $X_{t}$ in the prediction step of the Kalman filter algorithm. Thus, in the notation introduced above, this best guess is denoted $X_{t \mid t-1}$ and the approximation is given by

$$
g\left(X_{t} ; t_{0}^{i}, \tau^{i}, \psi\right) \approx g\left(X_{t \mid t-1} ; t_{0}^{i}, \tau^{i}, \psi\right)+\left.\frac{\partial g\left(X_{t} ; t_{0}^{i}, \tau^{i}, \psi\right)}{\partial X_{t}}\right|_{X_{t}=X_{t \mid t-1}}\left(X_{t}-X_{t \mid t-1}\right)
$$

Thus, by defining

$$
A_{t}(\psi) \equiv g\left(X_{t \mid t-1} ; t_{0}^{i}, \tau^{i}, \psi\right)-\left.\frac{\partial g\left(X_{t} ; t_{0}^{i}, \tau^{i}, \psi\right)}{\partial X_{t}}\right|_{X_{t}=X_{t \mid t-1}} X_{t \mid t-1} \quad \text { and }\left.\quad B_{t}(\psi) \equiv \frac{\partial g\left(X_{t} ; t_{0}^{i}, \tau^{i}, \psi\right)}{\partial X_{t}}\right|_{X_{t}=X_{t \mid t-1}}
$$

the measurement equation can be given on an affine form as

$$
\frac{\bar{P}_{t}^{i}\left(t_{0}^{i}, \tau^{i}\right)}{D_{t}^{i}\left(t_{0}^{i}, \tau^{i}\right)}=A_{t}(\psi)+B_{t}(\psi) X_{t}+\varepsilon_{t}^{i}
$$

and the steps in the algorithm proceed as previously described, except that the standard errors are obtained from

$$
\widehat{\Omega}^{Q M L}(\widehat{\psi})=\frac{1}{T} H(\widehat{\psi})^{-1}\left[\frac{1}{T} \sum_{t=1}^{T} \frac{\partial \log l_{t}(\widehat{\psi})}{\partial \psi} \frac{\partial \log l_{t}(\widehat{\psi})^{\prime}}{\partial \psi}\right]^{\prime} H(\widehat{\psi})^{-1},
$$

where $H(\psi)$.is the Hessian matrix evaluated as described in Harvey (1989). 


\section{Appendix D: The One-Step Approach and Full ML Estimation}

To describe the procedure for concentrating out $X_{1: T}$ of the log-likelihood function in equation (10), observe that

$$
\begin{aligned}
\frac{\partial L\left(\tilde{\psi} \mid Y_{1: T}\right)}{\partial X_{t}}= & -d_{t} R_{w}^{-1}\left(X_{t}-h\left(X_{t-1} ; \psi\right)\right) \\
& +h_{X}\left(X_{t} ; \psi\right)^{\prime} R_{w}^{-1}\left(X_{t+1}-h\left(X_{t} ; \psi\right)\right) \\
& -g_{X}\left(X_{t} ; \psi\right)^{\prime} R_{\varepsilon, t}^{-1}\left(Y_{t}-g\left(X_{t} ; \psi\right)\right) \\
= & 0
\end{aligned}
$$

for $t=1,2, \ldots, T$, where $d_{1}=0$ and $d_{t}=1$ for $t>1$. The matrix $h_{X}\left(X_{t} ; \psi\right) \equiv \frac{\partial h\left(X_{t} ; \psi\right)}{\partial X_{t}^{\prime}}$ and denotes the $n_{x} \times n_{x}$ Jacobian of $h\left(X_{t} ; \psi\right)$ with respect to $X_{t}$, and similarly, $g_{X}\left(X_{t} ; \psi\right) \equiv \frac{\partial g\left(X_{t} ; \psi\right)}{\partial X_{t}^{\prime}}$ with dimensions $n_{y, t} \times n_{x}$. Let $X_{1: T}^{(i)}$ denote the points around which the system in (8) and (9) is linearized. That is,

$$
\begin{gathered}
Y_{t}=g\left(X_{t}^{(i)} ; \psi\right)+g_{X}\left(X_{t}^{(i)} ; \psi\right)\left(X_{t}-X_{t}^{(i)}\right)+\varepsilon_{t} \\
X_{t+1}=h\left(X_{t}^{(i)} ; \psi\right)+h_{X}\left(X_{t}^{(i)} ; \psi\right)\left(X_{t}-X_{t}^{(i)}\right)+w_{t+1} .
\end{gathered}
$$

For a given value of $\psi$, let $\hat{X}_{1: T}(\psi)$ denote the state estimates from running the Kalman smoother on the linearized system in equations (14) and (15). As shown in Durbin and Koopman $(2001), \hat{X}_{1: T}(\psi)$ then solves the following system of equations

$$
\begin{aligned}
& -d_{t} R_{w}^{-1}\left(X_{t}-h\left(X_{t-1}^{(i)} ; \psi\right)-h_{X}\left(X_{t-1}^{(i)} ; \psi\right)\left(\hat{X}_{t-1}(\psi)-X_{t-1}^{(i)}\right)\right) \\
& +h_{X}\left(X_{t}^{(i)} ; \psi\right)^{\prime} R_{w}^{-1}\left(X_{t+1}-h\left(X_{t}^{(i)} ; \psi\right)-h_{X}\left(X_{t}^{(i)} ; \psi\right)\left(\hat{X}_{t}(\psi)-X_{t}^{(i)}\right)\right) \\
& -g_{X}\left(X_{t}^{(i)} ; \psi\right)^{\prime} R_{\varepsilon, t}^{-1}\left(Y_{t}-g\left(X_{t}^{(i)} ; \psi\right)-g_{X}\left(X_{t}^{(i)} ; \psi\right)\left(\hat{X}_{t}(\psi)-X_{t}^{(i)}\right)\right) \\
& =0
\end{aligned}
$$

for $t=1,2, \ldots, T$. Accordingly, when $\hat{X}_{t}(\psi)=X_{t}^{(i)}(\psi)$ for all $t$, the conditions in equations (16) reduces to those in equations (13), meaning that $\hat{X}_{1: T}(\psi)$ is the ML estimates of the states for a given value of $\psi$. The iterative procedure to find this solution is as follows:

Step 1 Run the Extended Kalman filter and smoother on the system in equations (8) and (9) to obtain $\hat{X}_{1: T}^{E K F}(\psi)$. Set $i=1$ and let $X_{1: T}^{(i)}(\psi)=\hat{X}_{1: T}^{E K F}(\psi)$.

Step 2 Run the Extended Kalman filter and smoother on the linearized system in equations (14) and (15) to obtain $\hat{X}_{1: T}(\psi)$.

Step 3 If $\left|\hat{X}_{t}(\psi)-X_{t}^{(i)}(\psi)\right|>\epsilon$ for any $t$, where $\epsilon$ is a small number, let $i=i+1$ and $X_{1: T}^{(i)}(\psi)=\hat{X}_{1: T}(\psi)$ and go to step 2, otherwise stop.

Let $\hat{X}_{1: T}(\psi)$ denote the states from this procedure, which depends on $\psi$. The concentrated log-likelihood function is then $L^{c}\left(\psi \mid Y_{1: T}\right) \equiv L\left(\psi, \hat{X}_{1: T}(\psi) \mid Y_{1: T}\right)$, which we optimize across $\psi$ to obtain the ML estimates. The asymptotic standard errors for $\hat{\psi}_{M L}$ are obtained in a standard fashion, i.e.,

$$
\widehat{A V a r}\left(\hat{\psi}_{M L}\right)=\left[\sum_{t=1}^{T} s_{i}^{c}\left(\hat{\psi}_{M L}\right) s_{i}^{c}\left(\hat{\psi}_{M L}\right)^{\prime}\right]^{-1}
$$

where the concentrated score $s_{i}^{c}\left(\hat{\psi}_{M L}\right)$ is computed by numerical differentiation of $L^{c}\left(\psi \mid Y_{1: T}\right)$. 


\section{Appendix E: Full ML Estimation with Stochastic Volatility}

Suppose $X_{t}$ evolves as

$$
X_{t+1}=h\left(X_{t} ; \theta\right)+f\left(X_{t}, \varepsilon_{t+1} ; \theta\right)
$$

where the $f$-function accommodates stochastic volatility in the dynamics of $X_{t}$. Lagging equation (18) by one period and inserting it into equation (8) gives

$$
\begin{aligned}
Y_{t} & =\underbrace{g\left(h\left(X_{t-1} ; \theta\right)+f\left(X_{t-1}, \varepsilon_{t}\right) ; \theta\right)}_{\tilde{g}\left(X_{t-1}, \varepsilon_{t} ; \theta\right)}+v_{t} \\
{\left[\begin{array}{c}
X_{t} \\
\varepsilon_{t+1}
\end{array}\right] } & =\left[\begin{array}{c}
h\left(X_{t-1} ; \theta\right)+f\left(X_{t-1}, \varepsilon_{t}\right) \\
0
\end{array}\right]+\left[\begin{array}{c}
0 \\
u_{t+1}
\end{array}\right]
\end{aligned}
$$

where $u_{t+1} \sim \mathcal{N} \mathcal{I D}\left(0, R_{\varepsilon}\right)$. Thus, by expanding the state vector to $\tilde{X}_{t}=\left[\begin{array}{cc}X_{t-1}^{\prime} & \varepsilon_{t}^{\prime}\end{array}\right]^{\prime}$, the law of motion in equation (18) with nonlinearities between the states and the innovations can be rewritten into an extended system with only linear innovations as in equations (8) and (9). 


\section{Appendix F: Formulas for Short Rate Expectations and Term Premiums}

In this appendix, we derive the analytical formulas for short-rate expectations and term premiums in the independent-factor AFNS model. Recall that term premium is defined as

$$
T P_{t}(\tau)=y_{t}(\tau)-\frac{1}{\tau} \int_{t}^{t+\tau} E_{t}^{P}\left[r_{s}\right] d s .
$$

Furthermore, recall that for affine stochastic differential equations of the form

$$
d X_{t}=K^{P}\left(\theta^{P}-X_{t}\right) d t+\Sigma d W_{t}^{P}
$$

the conditional expectation is given by

$$
E_{t}^{P}\left[X_{t+\tau}\right]=\left(I-\exp \left(-K^{P} \tau\right)\right) \theta^{P}+\exp \left(-K^{P} \tau\right) X_{t}
$$

In the AFNS model, the instantaneous short rate is defined as

$$
r_{t}=L_{t}+S_{t}
$$

while the specification of the $P$-dynamics considered is given by

$$
\left(\begin{array}{c}
d L_{t} \\
d S_{t} \\
d C_{t}
\end{array}\right)=\left(\begin{array}{ccc}
\kappa_{11}^{P} & 0 & 0 \\
0 & \kappa_{22}^{P} & 0 \\
0 & 0 & \kappa_{33}^{P}
\end{array}\right)\left[\left(\begin{array}{c}
\theta_{1}^{P} \\
\theta_{2}^{P} \\
\theta_{3}^{P}
\end{array}\right)-\left(\begin{array}{c}
L_{t} \\
S_{t} \\
C_{t}
\end{array}\right)\right] d t+\left(\begin{array}{ccc}
\sigma_{11} & 0 & 0 \\
0 & \sigma_{22} & 0 \\
0 & 0 & \sigma_{33}
\end{array}\right)\left(\begin{array}{c}
d W_{t}^{L, P} \\
d W_{t}^{S, P} \\
d W_{t}^{C, P}
\end{array}\right)
$$

Thus, the mean-reversion matrix is given by

$$
K^{P}=\left(\begin{array}{ccc}
\kappa_{11}^{P} & 0 & 0 \\
0 & \kappa_{22}^{P} & 0 \\
0 & 0 & \kappa_{33}^{P}
\end{array}\right)
$$

Its matrix exponential can be calculated analytically and is given by

$$
\exp \left(-K^{P} \tau\right)=\left(\begin{array}{ccc}
e^{-\kappa_{11}^{P} \tau} & 0 & 0 \\
0 & e^{-\kappa_{22}^{P} \tau} & 0 \\
0 & 0 & e^{-\kappa_{33}^{P} \tau}
\end{array}\right)
$$

Now, the conditional mean of the state variables is

$$
E_{t}^{P}\left[X_{t+\tau}\right]=\theta^{P}+\left(\begin{array}{ccc}
e^{-\kappa_{11}^{P} \tau} & 0 & 0 \\
0 & e^{-\kappa_{22}^{P} \tau} & 0 \\
0 & 0 & e^{-\kappa_{33}^{P} \tau}
\end{array}\right)\left(\begin{array}{c}
L_{t}-\theta_{1}^{P} \\
S_{t}-\theta_{2}^{P} \\
C_{t}-\theta_{3}^{P}
\end{array}\right)=\left(\begin{array}{c}
\theta_{1}^{P}+e^{-\kappa_{11}^{P} \tau}\left(L_{t}-\theta_{1}^{P}\right) \\
\theta_{2}^{P}+e^{-\kappa_{22}^{P} \tau}\left(S_{t}-\theta_{2}^{P}\right) \\
\theta_{3}^{P}+e^{-\kappa_{33}^{P} \tau}\left(C_{t}-\theta_{3}^{P}\right)
\end{array}\right)
$$

In order to get back to the term premium formula, we note that the conditional expectation of the instantaneous short rate process is:

$$
\begin{aligned}
E_{t}^{P}\left[r_{s}\right] & =E_{t}^{P}\left[L_{s}+S_{s}\right] \\
& =\theta_{1}^{P}+e^{-\kappa_{11}^{P}(s-t)}\left(L_{t}-\theta_{1}^{P}\right)+\theta_{2}^{P}+e^{-\kappa_{22}^{P}(s-t)}\left(S_{t}-\theta_{2}^{P}\right) .
\end{aligned}
$$

Next, we integrate the expected short rate over the time interval from $t$ to $t+\tau$ as in the definition of the term 
premium:

$$
\begin{aligned}
\int_{t}^{t+\tau} E_{t}^{P}\left[r_{s}\right] d s & =\int_{t}^{t+\tau}\left(\theta_{1}^{P}+e^{-\kappa_{11}^{P}(s-t)}\left(L_{t}-\theta_{1}^{P}\right)+\theta_{2}^{P}+e^{-\kappa_{22}^{P}(s-t)}\left(S_{t}-\theta_{2}^{P}\right)\right) d s \\
& =\left(\theta_{1}^{P}+\theta_{2}^{P}\right) \tau+\left(L_{t}-\theta_{1}^{P}\right) \frac{1-e^{-\kappa_{11}^{P} \tau}}{\kappa_{11}^{P}}+\left(S_{t}-\theta_{2}^{P}\right) \frac{1-e^{-\kappa_{22}^{P} \tau}}{\kappa_{22}^{P}} .
\end{aligned}
$$

The relevant term to go into the term premium formula is the average expected short rate

$$
\frac{1}{\tau} \int_{t}^{t+\tau} E_{t}^{P}\left[r_{s}\right] d s=\theta_{1}^{P}+\theta_{2}^{P}+\left(L_{t}-\theta_{1}^{P}\right) \frac{1-e^{-\kappa_{11}^{P} \tau}}{\kappa_{11}^{P} \tau}+\left(S_{t}-\theta_{2}^{P}\right) \frac{1-e^{-\kappa_{22}^{P} \tau}}{\kappa_{22}^{P} \tau} .
$$

The final expression for the term premium is then given by

$$
\begin{aligned}
T P_{t}(\tau)= & y_{t}(\tau)-\frac{1}{\tau} \int_{t}^{t+\tau} E_{t}^{P}\left[r_{s}\right] d s \\
= & L_{t}+\frac{1-e^{-\lambda \tau}}{\lambda \tau} S_{t}+\left(\frac{1-e^{-\lambda \tau}}{\lambda \tau}-e^{-\lambda \tau}\right) C_{t}-\frac{A(\tau)}{\tau} \\
& -\theta_{1}^{P}-\theta_{2}^{P}-\left(L_{t}-\theta_{1}^{P}\right) \frac{1-e^{-\kappa_{11}^{P} \tau}}{\kappa_{11}^{P} \tau}-\left(S_{t}-\theta_{2}^{P}\right) \frac{1-e^{-\kappa_{22}^{P} \tau}}{\kappa_{22}^{P} \tau} \\
= & \left(1-\frac{1-e^{-\kappa_{11}^{P} \tau}}{\kappa_{11}^{P} \tau}\right) L_{t}+\left(\frac{1-e^{-\lambda \tau}}{\lambda \tau}-\frac{1-e^{-\kappa_{22}^{P} \tau}}{\kappa_{22}^{P} \tau}\right) S_{t}+\left(\frac{1-e^{-\lambda \tau}}{\lambda \tau}-e^{-\lambda \tau}\right) C_{t} \\
& -\left(1-\frac{1-e^{-\kappa_{11}^{P} \tau}}{\kappa_{11}^{P} \tau}\right) \theta_{1}^{P}-\left(1-\frac{1-e^{-\kappa_{22}^{P} \tau}}{\kappa_{22}^{P} \tau}\right) \theta_{2}^{P}-\frac{A(\tau)}{\tau} .
\end{aligned}
$$




\section{References}

Andersen, Torben G., Nicola Fusari, and Viktor Todorov, 2015, "Parameter Inference and Dynamic State Recovery from Option Panels," Econometrica, Vol 83, No. 3, 1081-1145.

Andreasen, Martin M., 2013, "Non-Linear DSGE Models and The Central Difference Kalman Filter," Journal of Applied Econometrics, Vol. 28, 929-955.

Andreasen, Martin M. and Bent Jesper Christensen, 2015, "The SR Approach: A New Estimation Procedure for Non-Linear and Non-Gaussian Dynamic Term Structure Models," Journal of Econometrics, Vol. 184, No. 2, 420-451.

Andreasen, Martin M., Jens H. E. Christensen, and Simon Riddell, 2017, "The TIPS Liquidity Premium," Working Paper 2017-11, Federal Reserve Bank of San Francisco.

Bai, Jushan, 2003, "Inferential Theory for Factor Models of Large Dimensions," Econometrica, Vol. 71, No. 1, 135-171.

Bai, Jushan, and Serena Ng, 2002, "Determining the Number of Factors in Approximate Factor Models," Econometrica, Vol. 70, No. 1, 191-221.

Bauer, Michael D., Glenn D. Rudebusch, and Jing (Cynthia) Wu, 2012, "Correcting Estimation Bias in Dynamic Term Structure Models," Journal of Business and Economic Statistics, Vol. 30, No. 3, 454-467.

Björk, Tomas and Bent J. Christensen, 1999, "Interest Rate Dynamics and Consistent Forward Rate Curves," Mathematical Finance, Vol. 9, 323-348.

Black, Fisher, 1995, "Interest Rates as Options," Journal of Finance, Vol. 50, No. 7, 1371-1376.

Bliss, Robert R., 1996, "Testing Term Structure Estimation Methods," Advances in Futures and Options Research, Vol. 9, 197-231.

Bolder, David J., Grahame Johnson, and Adam Metzler, 2004, "An Empirical Analysis of the Canadian Term Structure of Zero-Coupon Interest Rates," Bank of Canada Working Paper No. 2004-48.

Bollerslev, Tim and Jeffrey M. Wooldridge, 1992, "Quasi-Maximum Likelihood Estimation and Inference in Dynamic Models with Time-Varying Covariances," Econometrics Reviews, Vol. 11, No. 2, 143-172.

Campbell, John Y. and Robert J. Shiller, 1991, "Yield Spread and Interest Rate Movements: A Bird's Eye View," Review of Economic Studies, Vol. 58, No. 3 , 495-514. 
Christensen, Jens H. E., Francis X. Diebold, and Glenn D. Rudebusch, 2009, "An ArbitrageFree Generalized Nelson-Siegel Term Structure Model," Econometrics Journal, Vol. 12, No. 3, C33-C64.

Christensen, Jens H. E., Francis X. Diebold, and Glenn D. Rudebusch, 2011, "The Affine Arbitrage-Free Class of Nelson-Siegel Term Structure Models," Journal of Econometrics, Vol. 164, No. 1, 4-20.

Christensen, Jens H. E. and Glenn D. Rudebusch, 2015, "Estimating Shadow-Rate Term Structure Models with Near-Zero Yields," Journal of Financial Econometrics, Vol. 13, No. 2, 226-259.

Christensen, Jens H. E. and Glenn D. Rudebusch, 2016, "Modeling Yields at the Zero Lower Bound: Are Shadow Rates the Solution?," in Eric Hillebrand, Siem Jan Koopman (ed.) Dynamic Factor Models (Advances in Econometrics, Volume 35) Emerald Group Publishing Limited, 75-125.

Dai, Qiang and Kenneth J. Singleton, 2000, "Specification Analysis of Affine Term Structure Models," Journal of Finance, Vol. 55, No. 5, 1943-1978.

Dai, Qiang, Kenneth J. Singleton, and Wei Yang, 2004, "Predictability of Bond Risk Premia and Affine Term Structure Models," Manuscript, Graduate School of Business, Stanford University.

Driessen, Joost, 2005, "Is Default Event Risk Priced in Corporate Bonds?," Review of Financial Studies, Vol. 18, No. 1, 165-195.

Duffee, Gregory R., 1999, "Estimating the Price of Default Risk," Review of Financial Studies, Vol. 12, No. 1, 197-226.

Duffee, Gregory R., 2002, "Term Premia and Interest Rate Forecasts in Affine Models," Journal of Finance, Vol. 57, No. 1, 405-443.

Durbin, J. and S. J. Koopman, 2001, "Time Series Analysis by State Space Methods," Oxford University Press, Oxford.

Fama, Eugene F., 1976, "Forward Rates as Predictors of Future Spot Rates," Journal of Financial Economics, Vol. 3, No. 4, 361-377.

Faria, Adriano and Caio Almeida, 2017, "A Hybrid Spline-Based Parametric Model for the Real Yield Curve," Manuscript, FGV Rio de Janeiro.

Filipović, Damir and Sander Willems, 2016, "Exact Smooth Term Structure Estimation," Manuscript, EPFL and Swiss Finance Institute. 
Fisher, Mark and Christian Gilles, 1996, "Term Premia in Exponential-Affine Models of the Term Structure," Manuscript, Board of Governors of the Federal Reserve System.

Fontaine, Jean-Sébastien and René Garcia, 2012, "Bond Liquidity Premia," Review of Financial Studies, Vol. 25, No. 4, 1207-1254.

Gürkaynak, Refet S., Brian Sack, and Jonathan H. Wright, 2007, "The U.S. Treasury Yield Curve: 1961 to the Present," Journal of Monetary Economics, Vol. 54, No. 8, 22912304 .

Gürkaynak, Refet S., Brian Sack, and Jonathan H. Wright, 2010, "The TIPS Yield Curve and Inflation Compensation," American Economic Journal: Macroeconomics, Vol. 2, No. $1,70-92$.

Hahn, Jinyong and Whitney Newey, 2004, "Jackknife and Analytical Bias Reduction for Nonlinear Panel Models," Econometrica, Vol. 72, No. 4, 1295-1319.

Harvey, A.C., 1989, Forecasting, structural time series models and the Kalman filter, Cambridge: Cambridge University Press.

Kim, Don H. and Kenneth J. Singleton, 2012, "Term Structure Models and the Zero Bound: An Empirical Investigation of Japanese Yields," Journal of Econometrics, Vol. 170, No. $1,32-49$.

Krippner, Leo, 2013, "A Tractable Framework for Zero Lower Bound Gaussian Term Structure Models," Discussion Paper 2013-02, Reserve Bank of New Zealand.

Nelson, Charles R. and Andrew F. Siegel, 1987, "Parsimonious Modeling of Yield Curves," Journal of Business, Vol. 60, No. 4, 473-489.

Pancost, N. Aaron, 2017, "Zero-Coupon Yields and the Cross-Section of Bond Prices," Chicago University, Working Paper, 1-66.

Steeley, James M., 2008, "Testing Term Structure Estimation Methods: Evidence from the UK STRIPs Market," Journal of Money, Credit and Banking, Vol. 40, No. 7, 1489-1512.

Svensson, Lars E. O., 1995, "Estimating Forward Interest Rates with the Extended NelsonSiegel Method," Quarterly Review, No. 3, Sveriges Riksbank, 13-26.

Swanson, Eric T. and John C. Williams, 2014, "Measuring the Effect of the Zero Lower Bound on Medium- and Longer-Term Interest Rates," American Economic Review, Vol. 104, No. 10, 3154-3185. 